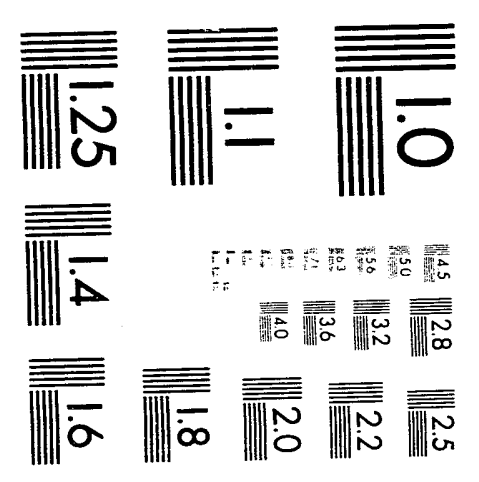



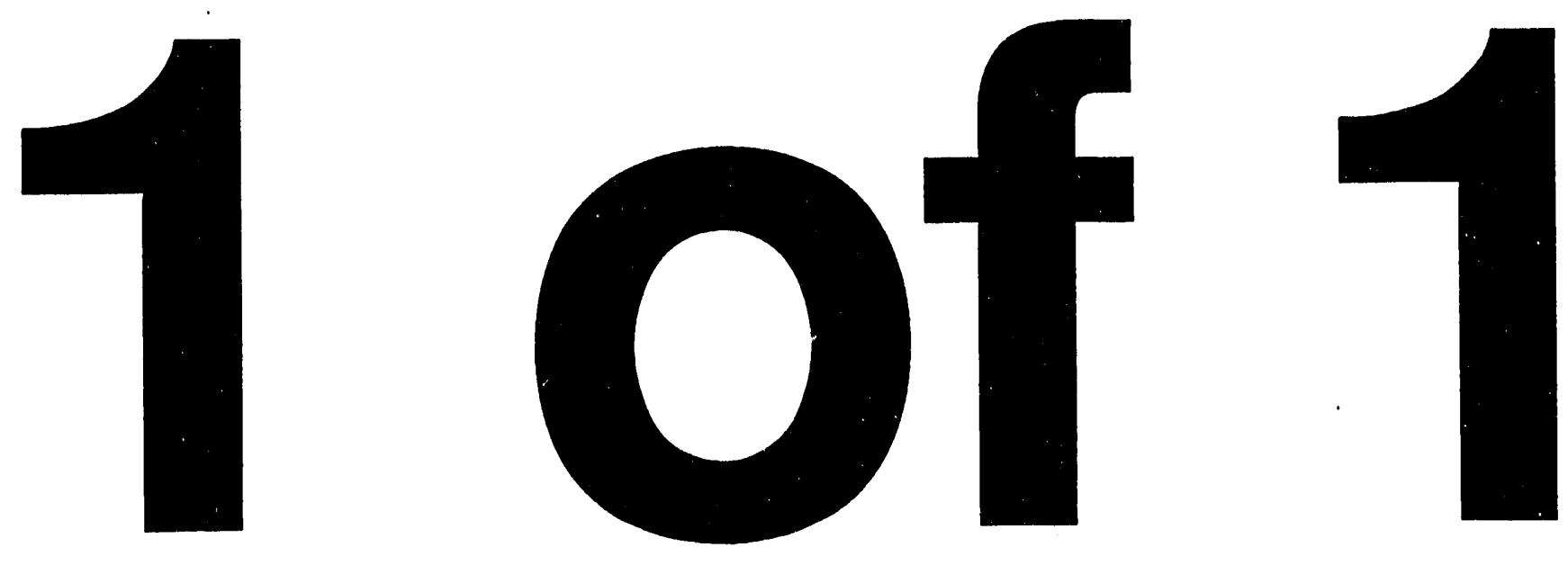


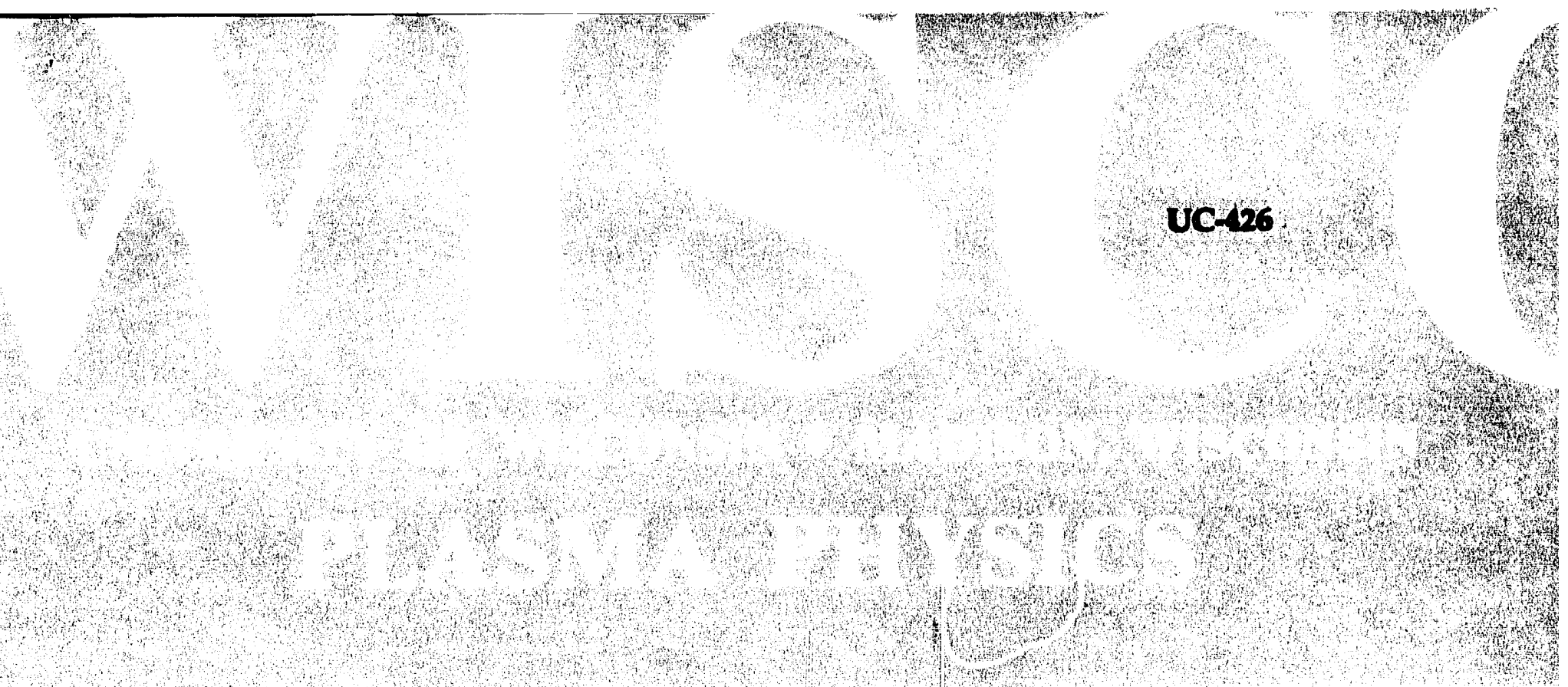

\section{CHAOS IN PLASMA SIMULATION AND EXPERIMENT}

Christopher Watts*, D.E. Newman+ and J.C. Sprott

DOE/ER/53198-223

September 1993

Fusion Resarch Center, Rollert L. Moore Building, Univereily of Tex2, Aüb, TX 78712

+Fusion Energy Division, MS. 8071, P.O Box 2009 , OAk Ridge National Laboratory, Oak Ridge, TN $37831-8076$
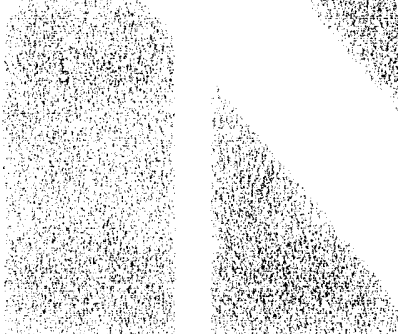


\section{NOTICE}

This report was prepared as an account of work sponsored by an agency of the United States Government. Neither the United States nor any agency thereof, nor any of their employees, makes any warranty, expressed or implied, or assumes any legal liability or responsibility for any third party's use or the results of such use of any information, apparatus, product or process disclosed in this report, or represents that its use by such third party would not infringe privately owned rights.

Printed in the United States of America

Available from

National Technical Information Service

U.S. Department of Commerce

5285 Port Royal Road

Springfield, VA 22161

NTIS Price codes

Printed copy: $\quad \mathrm{A} 03$

Microfiche copy: A01 


\title{
Chaos in Plasma Simulation and Experiment
}

\author{
Christopher Watts ${ }^{*}$, D. E. Newman ${ }^{\dagger}$ and J. C. Sprott \\ Department of Physics, University of Wisconsin - Madison, Madison, WI 53706
}

PACS numbers: $47.52,05.45,52.55, .52 .65, .06 .50$

\begin{abstract}
We investigate the possibility that chaos and simple determinism are governing the dynamics of reversed field pinch (RFP) plasmas using data from both numerical simulations and experiment. A large repertoire of nonlinear analysis techniques is used to identify low dimensional chaos. These tools include phase portraits and Poincaré sections, correlation dimension, the spectrum of Lyapunov exponents and short term predictability. In addition, nonlinear noise reduction techniques are applied to the experimental data in an attempt to extract any underlying deterministic dynamics. Two model systems are used to simulate the plasma dynamics. These are the DEBS code, which models global RFP dynamics, and the dissipative trapped electron mode (DTEM) model, which models drift wave turbulence. Data from both simulations show strong indications of low dimensional chaos and simple determinism. Experimental data were otitained from the Madison Symmetric Torus RFP and consist of a wide array of both global and local diagnostic signals. None of the signals shows any indication of low dimensional chaos or other simple determinism. Moreover, most of the analysis tools indicate the experimental system is very high dimensional with properties similar to noise. Nonlinear noise reduction is unsuccessful at extracting an underlying deterministic system.
\end{abstract}




\section{INTRODUCTION}

The search for evidence of chaos in plasmas has yielded mixed results. A wide range of chaotic processes has been reported, including the transition to turbulence and the formation of magnetic islands. ${ }^{1}$ Several groups have reported chaos and period-doubling behavior in glow discharges and pulsed machines. ${ }^{2-5}$ Low dimensional chaos has also been identified with drift wave turbulence both in experiment ${ }^{6}$ and simulations. ${ }^{7}$ In toroidal, fusion-caliber devices chaos has been reported in low frequency magnetic field oscillations in the DITE tokamak ${ }^{8}$ and in broadband $(0-100 \mathrm{kHz})$ magnetic field oscillations in the HBTX1A reversed field pinch (RFP). ${ }^{9}$ In the TFTR device low dimensional chaos has been reported in density fluctuations, with the dimension dependent on the wavenumber. ${ }^{10}$ In contrast, broadband magnetic and density fluctuations in the TCA tokamak show no evidence of low dimensional chaos. ${ }^{11}$

In this paper we examine the possibility that chaos or other simple determinism governs the dynamics of RFP discharges using data from both experiment and numerical simulations. Simulations suggest that chaos should be present in RFP discharges. However, it eludes all tests for identification in experimental data. As will be discussed, identification is particularly sensitive to the presence of noise and the stationarity of the system. This is additionally complicated by the fact that standard linear filtering techniques are not appropriate to signals generated by chaotic systems. Thus, although low dimensional chaos may govern MST discharges, it cannot be identified by the analysis techniques.

The Madison Symmetric Torus $(M S T)^{12}$ reversed field pinch provides a particularly good instrument for investigations in nonlinear dynamics in fusion plasmas. It is a large toroidal confinement device with discharges characterized by a spontaneous reversal of the toroidal magnetic field at the edge of the plasma. Although most measured quantities are characterized by broadband frequency spectra, most of the fluctuation power (ca. $90 \%$ ) is concentrated in a few long wavelength spatial modes. Experimental evidence and numerical simulations suggest that the mechanism behind these tearing mode fluctuations is a three wave coupling process, linking two $m=1$ modes to an $m=2$ mode. ${ }^{13}$ This corroboration of experiment and code indicates that the dominant plasma processes can be modeled as a deterministic system. 
The past decade has seen a tremendous effort towards correlating experimentally observed transport with computational models. Drift wave models in particular have shown promise towards explaining the anomalous ion heat loss in tokamaks. Previous studies of these models indicate that the dynamics are low dimensional, despite the large number of interacting modes in the system. ${ }^{7}$ The applicability of drift wave models to RFP transport is more tenuous. However, the models have sufficiently general nonlinear dynamics that many of the results should be relevant to RFP physics.

The paper is organized as follows: Section two reviews the techniques to be used in our analysis of the data. Section three presents the results from analysis of two numerical simulations. The DEBS code simulates RFP plasmas, and is thought to model correctly the dominant tearing mode fluctuations. The dissipative trapped electron mode (DTEM) model is designed to model drift wave turbulence thought to be a major contributor to transport in the core of tokamaks. Section four presents the results of analysis of experimental data from the MST. In the final section we discuss the results.

\section{ANALYSIS METHODS}

To ascertain whether low dimensional chaos governs RFP discharges, several of analysis techniques are used. These include calculation of the correlation dimension and the spectrum of Lyapunov exponents and assessing the short term predictability. Analysis is also performed on surrogate data records to guard against spurious determinations of low dimensional chaos. In addition, nonlinear noise reduction techniques are applied to the experimental data in an attempt to detect a low dimensional system in noise corrupted data. The methods used will be outlined in this section.

In general, one does not have access to all the independent variables of the system, and for experimental data, it may not be clear which variables are independent. Takens has proven that for dynamical systems, a single variable will capture all the relevant dynamics of the system subject to certain conditions. ${ }^{14}$ Specifically, given a system whose trajectory $F(t)=[x(t), y(t), z(t), \ldots]$ lies on an attractor there is a differentiable, reversible mapping from the manifold containing the attractor to that containing the attractor in delayed coordinates $X(t)=[\mid x(t), x(t+\tau), x(t+2 \tau), \ldots, x(t+m \tau)]$, so long as $m \geq 2 d+1$, where $d$ is the 
dimension containing the original attractor and $m$ is the dimension of the embedding space. The necessity of $2 d+1$ components is to insure that the vectors span the embedding space. Values of $m$ less than $2 d+1$ often suffice for proper reconstruction of the system.

Critical in implementing all analysis prncedures outlined in this section is the proper choice of the time lag $\tau$. For a finite data record, the time lag must be chosen such that vector components are independent. Choosing $\tau$ too small can result in artificially high correlation, and conversely, because chaotic systems are characterized by sensitivity to initial conditions, too large a value of $\tau$ leads to extreme decorrelation. A good criterion is $\tau$ times the embedding dimension should be 2 to 3 times the $e$-folding time of the autocorrelation function. However, all analysis procedures are performed using several values of $\tau$ to find an optimal value. Typically, there is a range of window lengths $(m-1) \tau$ over which the quantity being measured can be accurately estimated. There is an excellent discussion in Albano, et al. ${ }^{15}$ as this applies to the correlation dimension.

Firllomore, ihe data should be sampled frequently enough so that the autocorrelation time is several time: str?: Inferenent sampling will yield data that are improperly correlated; sampling too often will confinn 4 !n Minatirs to boly a portion of the attractor. Best results for all analysis methods presented hore.:." obtained when the autocorrelation time is around 10 to 20 time steps.

\section{A. Correlation dimension}

We estimate the fractal dimension of the data using the correlation dimension of Grassberger and Proccacia. $^{16}$ It is defined by:

$$
D_{2}=\lim _{r \rightarrow 0} \frac{\log C(r)}{\log r}
$$

where

$$
C(r)=\lim _{N \rightarrow \infty} \frac{1}{N^{2}} \sum_{i \neq j} \theta\left(r-\left|x_{i}-x_{j}\right|\right)
$$

and $\theta(r)$ is the Heavyside function whose value is one for $r>0$, and zero otherwise, and $N$ is the number of points. The correlation dimension provides a lower bound on the Hausdorff (fractal) dimension. The two 
often agree closely, differing by no more than a few percent.

Typically, one computes the correlation integral for various radii $r$ and plots $\log C(r)$ vs. $\log r$. Over some region in $\log r$, known as the scaling region, the slope is constant, and the value of this slope is taken as the correlation dimension. The two point slope $d[\log C(r)] / d[\log r]$ versus $\log r$ is more informative. This yields a plateau at the value of the correlation dimension, allowing a better determination of the scaling region.

Several groups have estimated the number of points required for a reliable determination of the correlation dimension. Estimates range from several hundred ${ }^{17}$ to $42^{d}{ }^{18}$ Tsonis provides a more reasonable rule: $N_{\min } \propto 10^{2+0.4 d} \cdot{ }^{19}$ We use a criterion based on the length of the scaling region: For adequate confidence in a correlation dimension determination a scaling region should span at least a decade in $r{ }^{20}$

\section{B. Lyapunov exponents}

The Lyapunov exponents measure the average divergence and convergence of orbits in phase space. Nearby trajectories of chaotic systems will, on average, diverge or converge exponentially in time. For the one diwiensional continuous map $x(t)=f_{1}\left(x_{0}\right)$, with two initial conditions separated by $\delta x$ the average separation after a time $t$ is $\delta x_{t} \approx \delta x_{0} e^{\lambda t}$. Taking the limit of infinitesimally separated points and infinite time the characteristic or Lyapunov exponent $\lambda$ is defined by

$$
\lambda=\lim _{t \rightarrow \infty} \frac{1}{t} \lim _{\delta x_{0} \rightarrow 0} \ln \left|\frac{\delta x_{t}}{\delta x_{0}}\right|=\lim _{t \rightarrow \infty} \frac{1}{t} \ln \left|\frac{d f_{t}(x)}{d x}\right| .
$$

A positive largest Lyapunov exponent has become the standard definition of a chaotic system.

For systems of more than one dimension there is a spectrum of Lyapunov exponents where each exponent corresponds to one of the principle axes of an ellipsoid centered on the trajectory and characterizes the local expansion or contraction of the attractor. The orientation of this ellipsoid changes as the trajectory evolves in time. The spectrum of Lyapunov exponents is given by:

$$
\lambda^{(k)}=\lim _{t \rightarrow \infty} \frac{1}{t} \ln \left\|\frac{\partial f_{t}\left(x^{(k)}\right)}{\partial x^{(k)}}\right\|=\lim _{t \rightarrow \infty} \frac{1}{t} \ln \left\|J_{t}^{(k)}\right\|, k=1,2, \ldots d .
$$

where $\left\|J_{t}^{(i)}\right\|$ is the $k^{\text {th }}$ eigenvalue of the $d$ dimensional Jacobian after advancing the system a time $t$ (see 
[21] for a thorough discussion).

Any continuous chaotic system must have at least three dimensions and hence three Lyapunov exponents. If the system is dissipative their sum must be negative to reflect the contraction of phase space. The least negative exponent controls the rate at which perturbed trajectories approach the inertial manifold. ${ }^{22} \mathrm{~A}$ chaotic system has a positive largest exponent, and any system continuous in time must have at least one Lyapunov exponent equal to zero corresponding to the lack of divergence (on average) tangent to the flow. ${ }^{23}$ Thus a three dimensional chaotic system has Lyapunov exponents $(+, 0,-)$.

Wolf, et al. ${ }^{22}$ have developed a method for calculating the largest exponent from the time series of a single variable of the chaotic system. The method follows a fiducial trajectory of the system and calculates the average separation of neighboring trajectories as they evolve. Although the procedure is effective, it is incomplete. A single Lyapunov exponent can only indicate that a system is chaotic, but tells nothing about the dimension of the system. Noise also has a positive largest Lyapunov exponent. One needs the entire spectrum of exponents to ascertain whether simple determinism exists.

A method proposed by Eckmann, et al..$^{24}$ and modified by Briggs ${ }^{25}$ computes the spectrum of exponents by approximating the Jacobian of the local trajectory of the dynamical system. In the method of Briggs, the time series is embedded in delay-space, a group of nearest neighbors is found, and their trajectory is fit to a polynomial. This function can then be differentiated analytically to obtain the Jacobian of the local dynamics.

Finding the eigenvalues of real, nonsymmetric matrices is not trivial, and often the eigenvalues are complex, whereas Lyapunov exponents are real. This is because the tangent space of the system is not necessarily aligned with our (arbitrary) coordinate system. Computation of the Lyapunov exponents is facilitated by $Q R$ decomposition. Any matrix can be written as $\mathbf{M}=\mathbf{Q R}$ where $\mathbf{Q}$ is an orthogonal matrix and $\mathbf{R}$ is upper right triangular with positive diagonal elements. This is known as the "skinny" decomposition, and it is unique. Since the time series to be analyzed is descretized, the Jacobian, using the chain rule, can be written as: $\mathbf{J}_{t+1}=\left[\partial f_{t+1} / \partial x\right]=\mathbf{J}_{t} \mathbf{J}_{t-1} \ldots \mathbf{J}_{1}=\mathbf{Q}_{(t)} \mathbf{R}_{(t)} \ldots \mathbf{R}_{(2)} \mathbf{R}_{(1)}$. The effect of the products $\mathbf{J} \mathbf{0}$ is to successively orient the Jacobian matrices along the principal axes of the tangent space of the attractor. The 
Lyapunov exponents are then given by:

$$
\lambda_{i}=\frac{1}{\tau n} \sum_{j=1}^{n} \ln R_{i i(j)}
$$

Implementation of the algorithm is not difficult on a computer, and several routines exist to do the $Q R$ decomposition. ${ }^{26,}{ }^{27}$ Most of the CPU time is spent searching for nearest neighbors rather than in actual calculation of the exponent. The Briggs method gives superior results to the original method proposed by Eckmann and Ruelle, because in fitting an arbitrary polynomial it includes the curvature of the local space. This is particularly relevant in embedding dimensions higher than the spatial dimension of the attractor. Kaplan and Yorke ${ }^{28}$ have conjectured a general formula which relates the Lyapunov exponents to the fractal dimension, $D_{0}$ :

$$
D_{\mathrm{KY}}=j+\frac{\sum_{i=1}^{j} \lambda_{i}}{\left|\lambda_{j+1}\right|} \approx D_{0} .
$$

$D_{\mathrm{KY}}$ is the Kaplan-Yorke or Lyapunov dimension. The Lyapunov exponents are ordered such that $\lambda_{i}>\lambda_{l+1}$. and $j$ is the largest index for which $\sum_{i=1}^{j} \lambda_{i}>0$. The conjecture appears to hold rigorously only for homogeneous attractors. However, it does hold approximately for many cases.

In calculating the spectrum of exponents, there are several test that the system is deterministic and not random. First, in higher embedding spaces the values of the exponents will remain reasonably constant, and additional exponents will be negative. A direct consequence of this is that the Kaplan-Yorke dimension saturates to a fairly constant value. In addition, continuous systems will have an exponent close to zero. Randomness can be identified by several characteristics. The data fill the space they are embedded in, as evidenced by the $\mathrm{KY}$ dimension and, although there are negative exponents, no convincing zero exponent exists. Negative exponents reflect the fact that the system is bounded. Moreover, it is difficult to find sufficient nearest neighbors, even for rather large radii because random points are sparsely and evenly distributed in the embedding space. 


\section{Nonlinear prediction}

One goal of identifying and characterizing chaotic behavior in experimental data is to develop a set of model equations for the system. A less ambitious goal is simply to predict the short-term behavior without knowing the governing equations explicitly. With this goal in mind, several methods have been developed, all of which use essentially the same procedure, with more or less sophistication. ${ }^{29-31}$ The time series is first embedded in an appropriate space using the method of time lags. The initial point for prediction is chosen, and the space is searched for its nearest neighbors. In the simplest method, the average of the neighbor's short-term trajectory becomes the basis for predicting the evolution of the initial point. The neighbors of this new point are then found, and the process is repeated. A superior method fits a linear or higher order polynomial to the local trajectory. We use a method similar to one proposed by Farmer and Sidorowich ${ }^{29}$ except that a generalized polynomial is fit to the local trajectory. The generalized polynomial can be written as

$$
F_{i}(x)=f_{i 0}+\sum f_{i 1} x+\sum f_{i 2} x x+\ldots .
$$

$F_{i}(x)$ is the map of the $i^{\text {th }}$ component and the sums are over all components in the embedding space $\left(f_{i} 1\right.$ is an $m$ dimensional vector, $f_{i 2}$ is an $m \times m$ tensor, etc.).

Tos measure the predictability we compute the average deviation of the predicted from the actual values, normalized to the distance propagated.

$$
e_{\text {predict }}=\frac{|y-x|}{|x|} .
$$

where $y$ is a vector pointing along the predicted orbit and $x$ points along the actual orbit. Because a single poor prediction (say in a sparsely populated region of phase space) can strongly skew the mean, the median is a better measure of the average normalized deviation. Predictions are done at successive time steps measured in units of $\tau-1$ for two reasons. Colored noise systems, particularly lowpass filtered noise, can give very small prediction errors if the time step is too small. Choosing the time step equal to $\tau$, however, will result in spurious correlations because components are shared among successive vectors. Hence the choice of $\tau-1$. Despite this choice, correlated noise (random systems with short term correlation, e.g. 
colored noise) will show some short-term predictability. White noise consistently has $100 \%$ error.

\section{Surrogate data sets}

Once a chaotic system has been identified, one needs to confirm that the identification is not an artifact of the analysis procedure. One method for doing so involves the creation of surrogate data sets. A surrogate set is similar to the original data, but randomized in some way to destroy the determinism. The analysis methods should then be reapplied to this new data set. All tests should yield negative results, thereby confirming the original assessment of determinism. If there still remain indications of determinism, one is likely witnessing an artifact of the analysis method.

The most effective method of creating a surrogate involves using the data from the original system and randomizing the phases of the Fourier components. This preserves the original power spectrum, but creates a data set in which small-scale spatial structure is lost. The method preserves global quantities of the system: the power spectrum (obviously), macrostructure in all embeddings (similar periodicily and amplitude) and also the autocorrelation time. Yet any simple determinism is destroyed.

\section{E. Nonlinear noise reduction}

While simple lowpass filtering may be appropriate in some cases, in general it is an inferior method of noise reduction. This is because the it does not take into account the inherent nonlinear dynamics of the system. The method simply attenuates all signal components greater than a certain frequency. This may, however, alter the relevant dynamics of the system, while ignoring other spurious components at lower frequencies. In addition, linear filtering methods can distort the phase information. As discussed in the previous section, this information is crucial to the dynamics of chaotic trajectories.

Several similar alternative methods of filtering specifically aimed at reducing noise in chaotic systems have been developed. ${ }^{32-38}$ The methods make use of the spatial structure of the underlying attractor and are similar in implementation to the prediction methods outlined in section II.C. The time record of a single variable of the system is embedded using the method of lags. For each point in the embedding space the nearest neighbors are found. The average local trajectory of these points is found which becomes the 
functional map of the point being filtered.

The method as outlined lacks self-consistency, however. One begins with a single time record and creates from it an array of time records, one corresponding to each dimension of the embedding space. The method for correcting this distinguishes the various approaches. One approach simply updates only a few of the components in the embedded space. For a given point in the embedded space $[x(t), x(t+\tau), x(t+2 \tau), \ldots]$, the filtered image point is $[y(t+\Delta t), y(t+\Delta t+\tau), \ldots x(t+\Delta t+n \tau), \ldots]$, where $y$ is the filtered signal. Thus, each new basis vector consists of some components from the filtered trajectory and the remaining components from the original signal. Our investigations indicate that updating only one, the leading component, yields the best results. The method is iterative, with several iterations required for best results. It achieves very good results for sets of ODEs with modest levels of additive noise $(<100 \%)$, recovering the original trajectory very nearly. The global deterministic properties of the system - fractal dimension, Lyapunov spectrum and short term predictability - are only partially recovered, since some small scale structure is lost. However, it is sufficient to be able to recognize the system as low dimensional.

More sophisticated methods to achieve self consistency use a window of points and find the best fit trajectory using a least squares method. ${ }^{32,37}$ Our investigations indicate results are not much better than the above method.

\section{NUMERICAL SIMULATIONS OF PLASMAS}

In this section we present results from two numerical plasma simulations. The first is a code that models global RFP dynamics. The second is a model of drift wave turbulence. Both systems show strong evidence of low dimensional chaos.

\section{A. DEBS code}

The DEBS code is a 3 dimensional magnetohydrodynamic (MHD) numerical simulation which with proper initialization will model reversed field pinch (RFP) discharges. ${ }^{39-42}$ The model has helped considerably in understanding tearing mode fluctuations in RFP plasmas. The agreement between experiment and simulation lends credibility to the code's ability to model real plasma processes. 
Although the code correctly models these phenomenon, there are some limitations. The model is pressureless, and thus does not include pressure driven modes. In addition, to have a reasonable computation time, the model uses a rather small value for the magnetic Reynolds number, or Lundquist number $S=\tau_{r} / \tau_{A} \cdot \tau_{r}$ is the resistive diffusion time, given by $\tau_{r}=4 \pi a^{2} / c^{2} \eta$, where $a$ is the scale size of the system and $\eta$ is the plasma resistivity. $\tau_{A}$ is the Alfvén time and is equal to $a$ divided by the Alfvén speed $v_{A}=B_{0} / \sqrt{4 \pi \rho_{0}}$ where $\rho_{0}$ is the density. Larger values of $S$ increase the computation time significantly because the system becomes more turbulent and requires a smaller time step size to accurately follow the wave dynamics. In the simulation presented here, the Lundquist number was $6 \times 10^{3}$, whereas the experiment is estimated to be the order of $10^{6}$. Despite this low value, several tens of CRAY CPU hours are required for each run to obtain a time record of sufficient length.

The code solves a reduced set of MHD equations via the semi-implicit method. This method allows relatively long time steps to track the relevant nonlinear phenomena by modifying the evolution of the destabilizing fast time-scale Alfvén modes. Details of the method can be found in the references. The dimensionless equations solved are:

$$
\begin{aligned}
\frac{\partial A}{\partial t} & =S V \times B-\eta J \\
\rho \frac{\partial V}{\partial t} & =-S \rho V \cdot \nabla V+S J \times B+v \nabla^{2} V
\end{aligned}
$$

The magnetic field $B$ is in units of the characteristic field strength $B_{0} . V$ is the fluid velocity in units of the Alfvén speed. $A$ is the vector potential, $J$ is the current density and $\rho$ is the mass density in units of $\rho_{0}$. Finally, $v$ is the viscosity coefficient $v=v_{0} \tau_{r} / a^{2}$, where $v_{0}$ is the characteristic viscosity. Both the viscosity and the mass density are assumed to be spatially constant; furthermore, the mass density is not evolved. The equations are solved in cylindrical geometry with periodic boundary conditions in the $z$ direction to mimic a torus.

For the data presented here the equations were solved for 9 toroidal and 3 poloidal modes with 127 radial mesh points. The system was monitored at regular intervals during which nine quantities were recorded. These were the parallel electric field $E_{\|}$, the average electric field $E_{\text {ave }}=-S\langle V \times B\rangle$, and the ohmic electric 
field $E_{R}=\eta J$ at both the edge and the core, the average toroidal and poloidal magnetic fluctuations, $d B_{t}$ $I d t$ and $d B_{p} / d t$, and toroidal loop voltage $V_{l o o p}$. We obtained about 20,000 data points for each signal covering about 1.65 resistive diffusion times. Direct comparison with MST discharges is not possible because of the compressed time scales used in the code. However, by comparing tearing mode time scales one arrives at a duration corresponding to about $55 \mathrm{~ms}$ for a standard MST discharge.

Figure 1 shows the time history of the average poloidal magnetic field fluctuations. Time is measure in units of the resistive diffusion time $\tau_{r}$. The trace shows evidence of flux jumps - bursts of magnetic field energy - which are also characteristic of MST discharges. The power spectrum of the signal is broadband, indicating that the system is not periodic.

The correlation dimensions for $d B_{p} / d t$ and its phase randomized surrogate are shown in figure 2 . The original signal shows a long plateau region with a value independent of embedding dimension. The surrogate shows a short region of spuriously low dimension which is similar to the behavior seen in correlated noise. Most other signals, including $E_{\|}$, and $E_{R}$ also have long plateaus of at least a decade at a dimension between three and four.

The prediction errors for $d B_{p} / d t, E_{R}$ and their surrogates are plotted in figure 3 . Both signals show fair predictability, better by a factor of three than their corresponding phase-randomized surrogates. However, an embedding greater than 18 is required before any evidence of short term predictability is seen. This behavior is odd considering that the dimension of the system is no greater than 5 , requiring an embedding of at most 11 to reconstruct the attractor faithfully. This may be attributable to the small number of data points.

Table I shows the Lyapunov exponents calculated for two of the representative quantities. In both cases, the Kaplan-Yorke dimension asymptotes to a constant value, and the Lyapunov exponents remain relatively constant as the embedding dimension increases. Both systems show a clear zero exponent.

\section{B. Dissipative trapped electron mode (DTEM) model}

A topic of intense research in the fusion plasma community is anomalous particle and energy transport. In 
tokamaks, one mechanism possibly responsible for this transport is the long-wavelength drift wave turbulence associated with dissipative trapped ion and electron modes. The existence of these modes is indicated by several experiments, ${ }^{43-45}$ although their link to confinement is still speculative. The specific model is probably not directly applicable to RFP physics. However, the basic structure of the model provides an instructive archetype for long wavelength turbulence in general. In particular, one of the nonlinearities, the polarization drift nonlinearity, has a direct correspondence to one of the MHD nonlinearities. Thus, although the model differs in the details, some of the global properties - saturation, spectral distribution of energy and transport - should be similar to those seen in RFP devices.

The DTEM model possesses two nonlinearities that govern its behavior. These are the $E \times B$ nonlinearity and the polarization-drift nonlinearity. A similar model used to study $\eta_{i}$ turbulence has been investigated for low dimensional chaos in [7]. The DTEM model equation is given by

$$
\frac{\partial \tilde{n}}{\partial t}+v^{*} \frac{\partial \tilde{n}}{\partial y}+\gamma \tilde{n}+D \frac{\partial^{2} \tilde{n}}{\partial y^{2}}-D L \nabla \frac{\partial \tilde{n}}{\partial y} \times z \cdot \nabla \tilde{n}+\rho c_{s} \nabla \tilde{n} \times z \cdot \nabla \rho^{2} \nabla^{2} \tilde{n}+\mu \nabla^{4} \tilde{n}=0,
$$

where $\tilde{n}$ is the fluctuating ion density, $v^{*}$ is the diamagnetic drift wave velocity $\left(c T_{e} / e B\right) / L, L$ is a density gradient scale length, $\gamma$ is a long wavelength collisional damping coefficient, $\rho$ is the ion gyroradius evaluated at the electron temperature and $c_{S}$ is the ion sound speed. $D$ is a negative diffusivity describing the destabilization of DTEM modes with $D=\varepsilon^{3 / 2} v^{* 2}\left(1+\frac{3}{2} \eta\right) / \gamma_{e}$ where $\gamma_{e}$ is the electron collisional damping coefficient and $\eta=d \ln T / d \ln n$ is the electron temperature gradient parameter. Finally, $\mu$ is the coefficient of hyper-viscosity introduced to model strong damping at short wavelength. The important nonlinear terms are the $5^{\text {th }}$ which is the $E \times B$ nonlinearity and the $6^{\text {th }}$, which is the polarization drift nonlinearity.

The code is purely spectral, meaning all computations are done in Fourier space. The system is initialized with a mode spectrum symmetric about the origin and allowed to evolve until transient behavior has vanisined. The magnetic field is oriented along the $z$ axis, and the density gradient is in the $x$ direction. In the jargon of the code, a $13 \times 13$ case contains the modes 0 though \pm 6 in both the $k x$ and $k y$ directions, denoted by $(k y, k x)$. For the work presented here the system was driven with a linear term (the 4 th term) at 
long wavelengths and damped at short wavelengths. The intermediate wavelength range of no damping or driving is known as the inertial range. Nondriven/nondamped systems were examined and appear also to be chaotic, however we will concentrate here on the more physical model. Several cases were examined, including $13 \times 13,21 \times 21$ and $29 \times 29$. We present here the results for the $21 \times 21$ case, which are representative. The data analyzed include the fluctuating energy from each of the modes in the inertial and damped regimes and the total fluctuating energy and enstrophy (mean squared vorticity). Results presented below indicate low dimensional chaos and simple determinism in most cases examined with a dimension dependent on the regime.

Figure 4 shows a typical time trace of the $(0,8)$ mode energy. This mode is part of the damped regime. Figure 5 shows the correlation dimension for the $(0,6)$ mode, part of the inertial range, and its phase randomized surrogate. There is a plateau region at a dimension near 6 which is not evident in the surrogate plot. The divot seen in the surrogate plot near a dimension of 7 is typical of the spurious plateau seen with correlated noise. Modes in the damped regime also indicate low dimensional dynamics, with a dimension near nine. The measured dimension of the inertial range is typically lower than that of the damped regime, however no strong dependence with mode number is seen for modes in the same regime. This behavior is not unreasonable and is consistent with the results reported in [7]: the regime in which the linear terms dominate (damping regime) shows a higher dimension than does the regime in which nonlinear terms dominate (inertial range).

Figure 6 demonstrates the short term predictability of the signals. Plotted are the prediction errors from the $(0,6)$ mode, the $(0,8)$ mode and the total energy along with the error of their phase-randomized suriogates. The short-term predictability of the $(0,8)$ mode is especially good: at least an order of magnitude better than its surrogate. The predictability of the energy is also good, though not quite as dramatic as the $(0,8)$ mode. The predictability of the $(0,6)$ mode is very poor, though, showing a high prediction error comparable to its surrogate's.

One should expect a system to have a single dimension characterizing its topological structure. We can offer some speculation as to why different modes show different dimensions and predictability. The DTEM 
model has two very different regimes, the inertial range and damped regime, which govern the dynamics of the system. In the inertial range the only dissipation is through the nonlinear coupling to the other modes, while the damping regime contains a dominant linear damping term. The dynamics of the damping regime are largely independent of the inertial range; the reverse, however, is not true. Thus, it may not be unreasonable that he two regimes have different dimensions when examining the large scale structure. At very small scale, this difference should disappear, reflecting the fact that it is coupled system. However, the number of points required and computational constraints restrict the ability to explore this limit of infinitesimal scale. Because the inertial range is very nearly Hamiltonian, trajectories are not strongly drawn to the attractor. Through 'he interaction with other modes, modes in this regime may experience perturbations from the inertial manifold of the attractor, and cannot quickly return to the manifold. In the damped regime, the dominant linear term insures that the trajectory remains close to the inertial manifold. accounting for its superior predictability.

Table II shows the Lyapunov exponents for the $(0,6)$ and $(0,8)$ modes in several embedding dimensions. Both systems have positive exponents, indicating that the modes are chaotic. The $(0,8)$ mode has a higher Kaplan-Yorke dimension before it saturates, as expected. The exponents are very stable from one embedding to the next, remaining at approximately the same value. This is a good indication that the values are probably very nearly correct. In addition, both show at least one and possibly two zero values.

\section{EXPERIMENTAL DATA FROM THE MADISON SYMMETRIC TORUS}

To ascertain whether simple determinism governs discharges in the Madison Symmetric Torus (MST) a wide range of signals was analyzed comprising both global and local measurements. Global signals included fluctuations in the average toroidal magnetic field at the wall, $d B_{t w} / d t$, fluctuations in the plasma current, $d I_{p} / d t$, chord averaged density fluctuations, both optical and soft $x$-ray radiation, and toroidal and poloidal gap voltages, $V_{t g}$ and $V_{p g}$. Local signals included local poloidal and toroidal magnetic field fluctuations, ion saturation current, $J_{\text {sat }}$, and plasma potential fluctuations. We present here a representative sample drawn from both groups.

The data were taken during the $40 \mathrm{~ms}$ flattop period of the MST discharge, which is the period over which 
the system is most nearly stationary. The global magnetic fields of a typical low current discharge $(\sim 290 \mathrm{kA})$ are shown in figure 7. Most signals were digitized at either 250 or $500 \mathrm{kHz}$, resulting in 10,000 to 20,000 points. The power spectra of all signals are broadband, with significant power in most up to $100 \mathrm{kHz}$. The autocorrelation times are extremely short, on the order of 2 to 3 time steps for all signals except $J_{s a t} . d B_{t w}$ Idt is plotted in figure 8. Evident in all signals are the flux jumps, bursts of magnetic field energy, which are characteristic of the MST discharges.

Figure 9 shows a comparison of the correlation dimension of $d B_{t w} / d t$ and its phase randomized surrogate. There is no saturation to a plateau with increasing embedding for any signal, indicating there is no evidence for low dimensional chaos or simple determinism in the unprocessed signals from MST. The curves of the original and surrogate data are indistinguishable, confirming that $d B_{t w} / d t$ is not a low dimensional chaotic process. Figure 10 depicts the prediction error for the MST signals. None of the signals are predictable in the short term. The steep rise from a modestly high value $(20-30 \%)$ to saturation at $100 \%$ within $2-3$ time steps is typical of correlated noise.

Table III presents the calculated Lyapunov exponents for the data. With each increase in embedding the Kaplan-York (KY) dimension increases significantly, although it does not equal the embedding dimension. Several of the signals, $J_{s a t} d B_{t} / d t$ and $d B_{p} / d t$, do show credible zero exponents, which would indicate the processes are continuous in time rather than random fluctuations. $d B_{t w} / d t$ and $d I_{p} / d t$ do not show a zero exponent. Because the KY dimension never saturates, the negative Lyapunov exponents cannot be trusted, despite the fact that some of the larger values appear relatively stationary. The positive values may be correct and reflect the space-filling property of the high dimensional system.

In addition to time-series analysis, MST discharges were analyzed spatially using a set of magnetic pickup coils distributed toroidaly around the torus. Rather than using time-delay embeddings to reconstruct the phase space, vectors were created by using signals from separate coils as individual vector components. The purpose was to address the issue of stationarity by analyzing data from a very short time period, and still have a sufficient number of vectors for adequate analysis. The correlation integral was computed using 2000 of these "spatial" vectors over a duration of $4 \mathrm{~ms}$ of the discharge in embeddings up to 9 . The number 
of coils corresponds to the embedding dimension. The results are consistent with the time series analysis: No evidence for simple determinism was seen in MST discharges.

Although there is no evidence for simple determinism in the raw data, the possibility remains that the signals are noise corrupted. Nonlinear noise reduction was applied to all signals in an attempt to extract an underlying low dimensional system. Figure 11 shows the effect of the filter on $d B_{t w} / d t$ after six iterations. Qualitatively, the two signals do not differ significantly from one another. The nonlinear noise reduction method is most effective if the time scale of the noise is significantly shorter than the time scale of the underlying dynamical system. These results indicate that this is not the case for MST signals.

The correlation dimension for $d B_{t w} / d t$ after noise reduction is plotted in figure 12 . There is still no evidence of low dimensional chaos in this or any of the data. Figure 13 shows the prediction error for the MST signals after nonlinear noise reduction. They actually show poorer predictive behavior than before filtering.

\section{v. CONCLUSIIDNS}

\section{A. Summary}

In this paper we examine the possibility that low dimensional chaos and simple determinism govern the dynamics of RFP plasma discharges using both experimental and numerical simulation data. In addition, several quantities have been measured to characterize fully the nonlinear dynamics.

Data from both numerical simulations show strong evidence for low dimensional chaos. The DEBS code, which models RFP discharges, shows a correlation dimension between 3 and 4 in both magnetic and electric field fluctuations. The spectrum of Lyapunov exponents gives a correspondingly low value for the Kaplan-Yorke dimension. Most signals indicate simple determinism with good short term predictability. Mode energies of the DTEM model, which simulates long-wavelength drift wave turbulence, also have a low correlation dimension, with a value near 6 for modes in the damped regime and near 9 for modes in the inertial range. Predictability is good for all cases, though better for modes in the damped regime than for those in the inertial range. The measured Lyapunov exponents corroborate these findings. 
Many local and global signals from the Madison Symmetric Torus were analyzed. Despite concerted effort, including the application of linear and nonlinear filtering techniques, no evidence of low dimensional chaos or simple determinism is found. There is no clear scaling region for the correlation integral, nor does the Kaplan-Yorke dimension saturate. All data have poor short-term predictability.

\section{B. Discussion}

The fact that the experimental data from the MST show no simple determinism must be contrasted with the positive results of both the numerical simulations and previous results from other experiments. The discrepancy between code and experiment has several likely origins. The DEBS code, although it seems to model some RFP processes well, contains several crucial simplifications. The model lacks temperature and pressure effects. Another simplification is the small number of modes. The 27 modes 9 toroidal by 3 poloidall used in the DEBS code is probably far too few to model the highly turbulent system of the experiment. The different Lundquist numbers also probably contributed to the difference between the experiment and simulation since the nonlinear interaction among the modes increases with Lundquist number. Finally, in order to efficiently model the long wavelength tearing modes of the plasma, the code does not treat fast fluctuations correctly. The broadband nature of the experimental data indicates that these high-frequency oscillations may be important to the system dynamics. One could reasonably expect that the dimension should increase significantly if some or all of these simplifications were rectified in the model.

That low dimensional chaos is seen in the DTEM model and not in the experimental data from MST may not be too disconcerting since drift wave turbulence is believed not to be significant in RFP plasmas. Yet the model is in many ways general enough that one might expect to see similar results despite this difference. The code suggests that one should see simple determinism in individual mode amplitudes. However, analysis of individual mode amplitudes from magnetic fluctuations in MST yielded negative results. The simulation models only one of several processes occurring simultaneously in a real nlasma. This omission could account for the difference.

There may be several obstacles that hinder the identification of low dimensional dynamics in the 
experiment. Apparent from the signals is that the magnetic flux jumps are a dominant feature. Yet the data records, due to the discharge length, contain only a few flux jumps. Typically, one requires several hundred cycle times of the "dominant" period of the system for proper astimation of the dimension. A related problem is that, if the system is high dimensional, many more points are needed to estimate that dimension.

A second, and probably more important concern, is the issue of stationarity. Even a very small random fluctuation in one of the system parameters can make the dimension immeasurable. One possible such process is the influx of impurity ions into the plasma. Non-random (deterministic) perturbations can increase the dimension by at most one for each fluctuating quantity. However, if several presumably stationary parameters (e.g. loop voltage or primary current) fluctuate this may put impractical limits on the number of points required to estimate the dimension.

On the basis of our analysis we must conclude that the dynamics of the RFP is probably high dimensional. When analyzing a system, even if all parameters - embedding delay time, embedding dimension, number of points, etc. - are not optimally determined thero is at least some indication of a plateau in the correlation dimension plots. The data from the MST show not the slightest hint of a plateau, even for embeddings up to 30. The behavior of the correlation plots is very similar to high dimensional or random data. We estimate that the dimension is probably greater than 15 , using the criterion in [19] with 20,000 points and a minimum plateau length of 0.2 decades in $r$. Our results make us skeptical of previously reported claims of low dimensionality in turbulent magnetoplasmas.

Acknowledaments: We would like to thank Keith Briggs for providing his code for computation of the Lyapunov exponent spectrum. This work is supported by the U.S. Department of Energy.

\footnotetext{
* Present address: Fusion Research Center, Robert L. Moore Bldg., University of Texas, Austin, TX 78712. email address: watts@juno.physics.wisc.edu. This article is based on work done in partial flufillment of the requirements for the degree of Ph.D.
} 
† Present address: Fusion Energy Division, M.S. 8071, P.0. Box 2009, Oak Ridge National Laboratory, Oak Ridge, TN $37831-8076$ 


\section{Tables}

\begin{tabular}{|l|c|c|ccc|}
\hline system & $\tau$ & $D_{K Y}$ & Lyapunov exponents & \\
\hline$d B_{D} l d t$ & 0.96 & 5.31 & $0.3738 \pm 0.012^{\prime}$ & $0.1247 \pm 0.0091$ & $0.0129 \pm 0.0099$ \\
& & $-0.0535 \pm 0.0112$ & $-0.2184 \pm 0.0177$ & $-0.7796 \pm 0.0343$ \\
& 0.88 & 5.85 & $0.3638 \pm 0.0106$ & $0.1341 \pm 0.0084$ & $0.0113 \pm 0.0085$ \\
& & & $-0.0535 \pm 0.0095$ & $-0.1520 \pm 0.0108$ & $-0.3555 \pm 0.0146$ \\
& & & $-0.9002 \pm 0.0288$ & & \\
\hline$E_{\text {ave }}$ & 0.96 & 6.30 & $0.3569 \pm 0.0111$ & $0.1537 \pm 0.0092$ & $0.0262 \pm 0.0083$ \\
& & & $-0.0033 \pm 0.0033$ & $-0.0760 \pm 0.0113$ & $-0.2296 \pm 0.014 ?$ \\
& & & $-0.7577 \pm 0.0265$ & & \\
\hline & 0.80 & 6.82 & $0.3680 \pm 0.0103$ & $0.1951 \pm 0.0086$ & $0.0404 \pm 0.0076$ \\
& & & $-0.0091 \pm 0.0082$ & $-0.0840 \pm 0.0094$ & $-0.1988 \pm 0.0109$ \\
& & & $-0.3791 \pm 0.0144$ & $-0.9597 \pm 0.0288$ & \\
\hline
\end{tabular}

Table I. The Lyapunov exponents and Kaplan-Yorke dimension for two of the quantities analyzed from DEBS code.

\begin{tabular}{|c|c|c|c|c|c|}
\hline system & $\tau$ & $D_{K Y}$ & \multicolumn{3}{|c|}{ Lyapunov exponents } \\
\hline \multirow[t]{7}{*}{ mode $(0,6)$} & $0.09 \mathrm{~s}$ & 8.10 & & $1.0174 \pm 0.0315$ & $0.5018 \pm 0.0276$ \\
\hline & & & $0.0950 \pm 0.0260$ & $-0.0222 \pm 0.0222$ & $.0 .4130 \pm 0.0335$ \\
\hline & & & $-0.9218 \pm 0.0396$ & $-1.3575 \pm 0.0564$ & $-3.8867 \pm 0.1088$ \\
\hline & $0.08 \mathrm{~s}$ & 8.55 & $1.7148 \pm 0.0377$ & $0.9704 \pm 0.0301$ & $0.4574 \pm 0.0261$ \\
\hline & & & $0.0757 \pm 0.0235$ & $.0 .0525 \pm 0.0268$ & $-0.2167 \pm 0.0290$ \\
\hline & & & $-0.6746 \pm 0.0337$ & $.1 .1945 \pm 0.0430$ & $-1.9802 \pm 0.0594$ \\
\hline & & & $-4.4591 \pm 0.1159$ & & \\
\hline \multirow[t]{8}{*}{ mode $(0,8)$} & $0.05 \mathrm{~s}$ & 9.09 & $8.8462 \pm 0.1415$ & $4.9430 \pm 0.1087$ & $2.5281 \pm 0.0860$ \\
\hline & & & $1.1518 \pm 0.0841$ & $0.0490 \pm 0.0490$ & $-0.3610 \pm 0.0996$ \\
\hline & & & $-1.2486 \pm 0.1249$ & $-3.9239 \pm 0.2864$ & $-9.2991 \pm 0.5115$ \\
\hline & & & $-29.022 \pm 1.073$ & & \\
\hline & $0.04 \mathrm{~s}$ & 9.63 & $8.9888 \pm 0.1169$ & $5.7238 \pm 0.1030$ & $3.3122 \pm 0.0894$ \\
\hline & & & $1.3845 \pm 0.0872$ & $0.1431 \pm 0.0976$ & $-0.4498 \pm 0.1183$ \\
\hline & & & $-1.9071 \pm 0.1354$ & $-3.7892 \pm 0.1629$ & $-6.5160 \pm 0.1955$ \\
\hline & & & $-10.868 \pm 0.250$ & $-23.434 \pm 0.398$ & \\
\hline
\end{tabular}

Table II. The Lyapunov exponents and Kaplan-Yorke dimension for two modes of the DTEM model. 


\begin{tabular}{|c|c|c|c|c|c|}
\hline signal & $\tau$ & $D_{K Y}$ & \multicolumn{3}{|c|}{ Lyapunov exponents } \\
\hline \multirow[t]{2}{*}{$d B_{t w} / d t$} & \multirow[t]{2}{*}{$2 \mu s$} & 4.02 & $\begin{array}{r}0.1570 \pm 0.0011 \\
-0.1790 \pm 0.0029 \\
\end{array}$ & $\begin{array}{c}0.0691 \pm 0.0012 \\
-0.5480 \pm 0.0049 \\
\end{array}$ & $-0.0342 \pm 0.0018$ \\
\hline & & 6.15 & $\begin{array}{l}0.1186 \pm 0.0008 \\
-0.0162 \pm 0.0012 \\
-0.2057 \pm 0.0029\end{array}$ & $\begin{array}{l}0.0701 \pm 0.0008 \\
-0.0569 \pm 0.0017 \\
-0.4776 \pm 0.0043\end{array}$ & $\begin{array}{l}0.0288 \pm 0.0009 \\
-0.1134 \pm 0.0023\end{array}$ \\
\hline \multirow[t]{2}{*}{$d l_{p} / d t$} & \multirow[t]{2}{*}{$2 \mu \mathrm{s}$} & 4.11 & $\begin{array}{r}0.1589 \pm 0.0011 \\
-0.1472 \pm 0.0026 \\
\end{array}$ & $\begin{array}{c}0.0670 \pm 0.0012 \\
-0.5068 \pm 0.0046 \\
\end{array}$ & $-0.0205 \pm 0.0016$ \\
\hline & & 6.24 & $\begin{array}{l}0.1227 \pm 0.0009 \\
0.0093 \pm 0.0011 \\
0.0000 \pm 0.0000\end{array}$ & $\begin{array}{c}0.0726 \pm 0.0008 \\
-0.0536 \pm 0.0016 \\
-0.4987 \pm 0.0045\end{array}$ & $\begin{array}{l}0.0296 \pm 0.0009 \\
-0.1100 \pm 0.0023\end{array}$ \\
\hline \multirow[t]{2}{*}{$\begin{array}{l}J_{\text {sat }} \\
\lambda / 10\end{array}$} & \multirow[t]{2}{*}{$10 \mu \mathrm{s}$} & 4.09 & $\begin{array}{l}0.0432 \pm 0.0021 \\
-0.0411 \pm 0.0032\end{array}$ & $\begin{array}{c}0.0159 \pm 0.0021 \\
-0.1047 \pm 0.0059\end{array}$ & $-0.0081 \pm 0.0024$ \\
\hline & & 5.07 & $\begin{array}{l}0.0509 \pm 0.0023 \\
-0.0181 \pm 0.0024 \\
\end{array}$ & $\begin{array}{r}0.0232 \pm 0.0018 \\
-0.0481 \pm 0.0035 \\
\end{array}$ & $\begin{array}{l}0.0019 \pm 0.0019 \\
-0.1427 \pm 0.0073 \\
\end{array}$ \\
\hline \multirow[t]{2}{*}{$d B_{p} / d t$} & \multirow[t]{2}{*}{$4 \mu \mathrm{s}$} & 4.04 & $\begin{array}{l}0.0670 \pm 0.0009 \\
-0.0636 \pm 0.0015\end{array}$ & $\begin{array}{l}0.0257 \pm 0.0009 \\
-0.1731 \pm 0.0028 \\
\end{array}$ & $-0.0149 \pm 0.0011$ \\
\hline & & 6.66 & $\begin{array}{l}0.0567 \pm 0.0009 \\
-0.0009 \pm 0.0007 \\
-0.0723 \pm 9.0014 \\
\end{array}$ & $\begin{array}{c}0.0342 \pm 0.0007 \\
-0.0174 \pm 0.0008 \\
-0.1751 \pm 0.0028 \\
\end{array}$ & $\begin{array}{l}0.0147 \pm 0.0006 \\
-0.0399 \pm 0.0010\end{array}$ \\
\hline \multirow[t]{2}{*}{$d B_{t} / d t$} & \multirow[t]{2}{*}{$4 \mu \mathrm{s}$} & 4.19 & $\begin{array}{r}0.0698 \pm 0.0010 \\
-0.0599 \pm 0.0016 \\
\end{array}$ & $\begin{array}{l}0.0317 \pm 0.0009 \\
-0.1645 \pm 0.0028\end{array}$ & $-0.0102 \pm 0.0011$ \\
\hline & & 6.77 & $\begin{array}{l}0.0581 \pm 0.0009 \\
0.0005 \pm 0.0005 \\
.0 .0718 \pm 0.0014\end{array}$ & $\begin{array}{l}0.0350 \pm 0.0007 \\
-0.0173 \pm 0.0008 \\
-0.1708 \pm 0.0027\end{array}$ & $\begin{array}{l}0.0169 \pm 0.0006 \\
-0.0377 \pm 0.0009\end{array}$ \\
\hline
\end{tabular}

Table III. Lyapunov exponents for the MST signals, measured in $\mu \mathrm{s}^{-1}$, and corresponding Kaplan-Yorke dimensions. 


\section{Figure Captions}

Figure 1. Fluctuations of the poloidal magnetic field at the wall from the DEBS code.

Figure 2. Correlation dimension for $d B_{p} / d t$ from the DEBS code and its phase randomized surrogate. The data show a scaling region not present in the surrogate.

Figure 3. Prediction error for the DEBS simulation. Predictability for bath $d B_{p} / d t$ and $E_{R}$ are better by a factor of three than their surrogates.

Figure 4. The energy of the $(0,6)$ mode of a $21 \times 21$ mode system in the DTEM model. This mode is in the damped regime.

Figure 5. The correlation dimension of the $(0,8)$ mode of DTEM model. There is a clear scaling region for the original signal at a dimension between 8 and 9 not evident in the surrogate.

Figure 6. The prediction error for the $(0,6),(0,8)$ modes and total energy of the $21 \times 21$ mode spectrum. Predictability is good for both the $(0,8)$ mode and the total energy, though poor for the $(0,6)$ mode.

Figure 7. The magnetic fields for a typical MST discharge. The data presented here were analyzed during the flat-top period from about 17-57 ms.

Figure 8. Fluctuations in the toroidal magnetic field at the wall in the MST.

Figure 9. Comparison of the correlation dimension of $d B_{t w} / d t$ in MST with its surrogate data set. Both show very similar behavior, indicating that $d B_{t w} / d t$ is not governed by a low dimensional chaotic process.

Figure 10. The prediction error for MST signals. None show any short term predictability beyond that of correlated noise.

Figure 11. The effect of nonlinear noise reduction on $d B_{t w} / d t$. The original and noise reduced signal are nearly identical. 
Figure 12. The correlation dimension of $d B_{t w} / d t$ from the MST signals after processing with the nonlinear noise reduction method. There are still no indications of low dimensional chaos.

Figure 13. Prediction error for MST signals after processing with the nonlinear filter. Predictability has deteriorated in most signals relative to the unfiltered case (cf. figure 10). 


\section{References}

${ }^{1}$ Horton, C.W. and Reichel, L., Statistical Physics and Chaos in Fusion Plasmas (John Wiley \& Sons, New York, 1984).

${ }^{2}$ Braun, T., Lisboa, J., and Gallas, J., Phys. Rev. Lett. 68, 2770 (1992).

${ }^{3}$ Oin, J., Wang, L., Yuan, D., Gao, P., and Zhang, B., Phys. Rev. Lett. 63, 163 (1989).

${ }^{4}$ Cheung, P. and Wong, A., Phys. Rev. Lett. 59 (1987).

${ }^{5}$ Chu, J.H. and I, L., Phys. Rev. A 39, 233 (1989).

${ }^{6}$ Strohlein, G. and Piel, A., Phys. Fluids B 1, 1168 (1989).

${ }^{7}$ Persson, M. and Nordman, H., Phys. Rev. Lett. 67, 3396 (1991).

${ }^{8}$ Arter, W. and Edwards, D.N., Phys. Lett. 114A, 84 (1986).

${ }^{9}$ Gee, S.J. and Taylor, J.B., in 12th European Conference on Controlled Fusion and Plasma Physics (September, 1985).

${ }^{10}$ Barkley, H.J., Andreoletti, J., Gervais, F., Olivain, J., Quemeneur, A., and A. Truc, Plasma Phys. Controlled Fusion 30, 217 (1988).

${ }^{11}$ Sawley, M.L., Simm, W., and Pochelon, A., Phys. Fluids 30, 129 (1987).

${ }^{12}$ Dexter, R.N., Kerst, D.W., Lovell, T.W., Prager, S.C., and Sprott, J.C., Fusion Technol. 19, 131 (1991).

${ }^{13}$ Assadi, S., Phys. Rev. Lett. 69, 281 (1992).

${ }^{14}$ Takens, F., in Dynamical Systems of Turbulence, Rand, D.A. and Young, L.S. ed. (Springer, Berlin, 1981), Lecture Notes in Mathematics, vol. 898, p. 366.

${ }^{15}$ Albano, A.M., Muench, J., and Schwartz, C., Phys. Rev. A 38, 3017 (1988).

${ }^{16}$ Grassberger, P. and Procaccia, I., Phys. Rev. Lett. 50, 346 (1983).

${ }^{17}$ Havstad, J.W. and Ehlers, C., Phys. Rev. A 39, 845 (1989). 
${ }^{18}$ Smith, L., Phys. Lett. A 133, 283 (1988).

${ }^{19}$ Tsonis, A., Chaos: From theory to applications (Plenum, New York, 1992).

${ }^{20}$ Ruelle, D., Proc. R. Soc. Lond. A, 241 (1990).

${ }^{21}$ Eckmann, J.P. and Ruelle, D., Rev. Mod. Phys. 57, 617 (1985).

${ }^{22}$ Wolf, A., Swift, J., Swinney, H., and Vastano, J., Physica D, 285 (1985).

${ }^{23}$ Haken, H., Phys. Lett. 94A, 71 (1983).

${ }^{24}$ Eckmann, J.P., Kamphorst, S.O., Ruelle, D., and Ciliberto, S., Phys. Rev. A 34, 4971 (1986).

${ }^{25}$ Briggs, K., Phys. Lett. A 151, 27 (1990).

26Wilkinson, J.H. and Reinsch, C., Linear Algebra (Springer, Berlin, 1971).

${ }^{27}$ LAPACK FORTRAN Routines, available by anonymous ftp from netlib@research.att.com.

${ }^{28}$ Kaplan, J. and Yorke, J., in Chaotic behavior of multidimensional difference equations, Peitgen, H. and W., H.W. ed. (Springer, 1979).

${ }^{29}$ Farmer, J.D. and Sidorowich, J., Phys. Rev. Lett. 59, 845 (1987).

${ }^{30}$ Sugihara, G. and May, R., Nature 344, 734 (1990).

${ }^{31}$ Wayland, R., Bromley, D., Pickett, D., and Passamante, A., Phys. Rev. Lett. 70, 580 (1993).

${ }^{32}$ Kostelich, E.J. and Yorke, J.A., Phys. Rev. A 38, 1649 (1988).

${ }^{33}$ Schreiber, T. and Grassberger, P., Phys. Lett. A 160, 411 (1991).

${ }^{34}$ Sauer, T., Physica D 58, 193 (1992).

${ }^{35}$ Cawley, R. and Hsu, G.H., Phys. Rev. A 46, 3057 (1992).

${ }^{36}$ Grassberger, P., Hegger, T., Kantz, H., Schaffrath, C., and Schreiber, T., Chaos 3, 127 (1993).

${ }^{37}$ Enge, N., Buzug, T., and Pfister, G., Phys. Lett. A 175, 178 (1993).

${ }^{38}$ Schreiber, T., Phys. Rev. E 47, 2401 (1993). 
${ }^{39}$ Harned, D.S. and Schnack, D.D., J. Comp. Phys. 65, 57 (1986).

${ }^{40}$ Kirby, P., Comp. Phys. Comm 47, 17 (1987).

${ }^{41}$ Schnack, D.D., Barnes, D.C., Mikic, Z., Harned, D.S., and Caramana, E.J., J. Comp. Phys. 70, 330 (1987).

${ }^{42}$ Ho, Y.L., Ph.D. thesis, UW-Madison, 1989.

${ }^{43}$ Fonck, R.J., Paul, S.F., Roberts, D.R., Kim, Y.J., Bretz, N., D. Johnson, R. Nazikian, and G. Taylor, in 18th European conference of controlled fusion and plasma physics, Bachmann, P. and Robinson, D.C. ed., vol. 15C (Berlin, 1991) p. I-269.

${ }^{44}$ Cripwell, P. and Costley, A.E., in 18th European conference of controlled fusion and plasma physics, Bachmann, P. and Robinson, D.C. ed., vol. 15C (Berlin, 1991) p. I-17.

${ }^{45}$ Brower, D.L., Peebles, W.A., and Luhmann, N.C., Nucl. Fusion 27, 2055 (1987). 

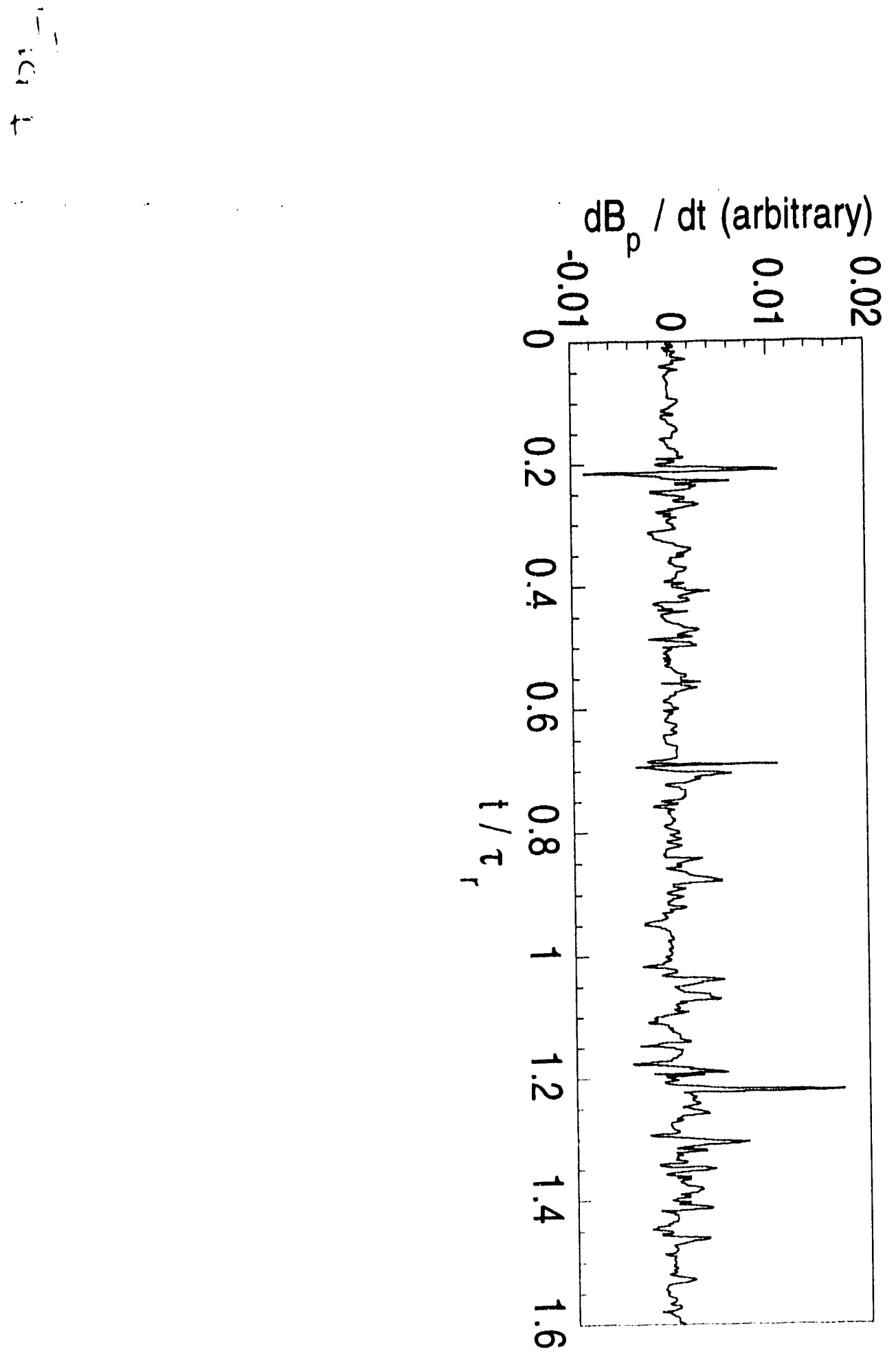


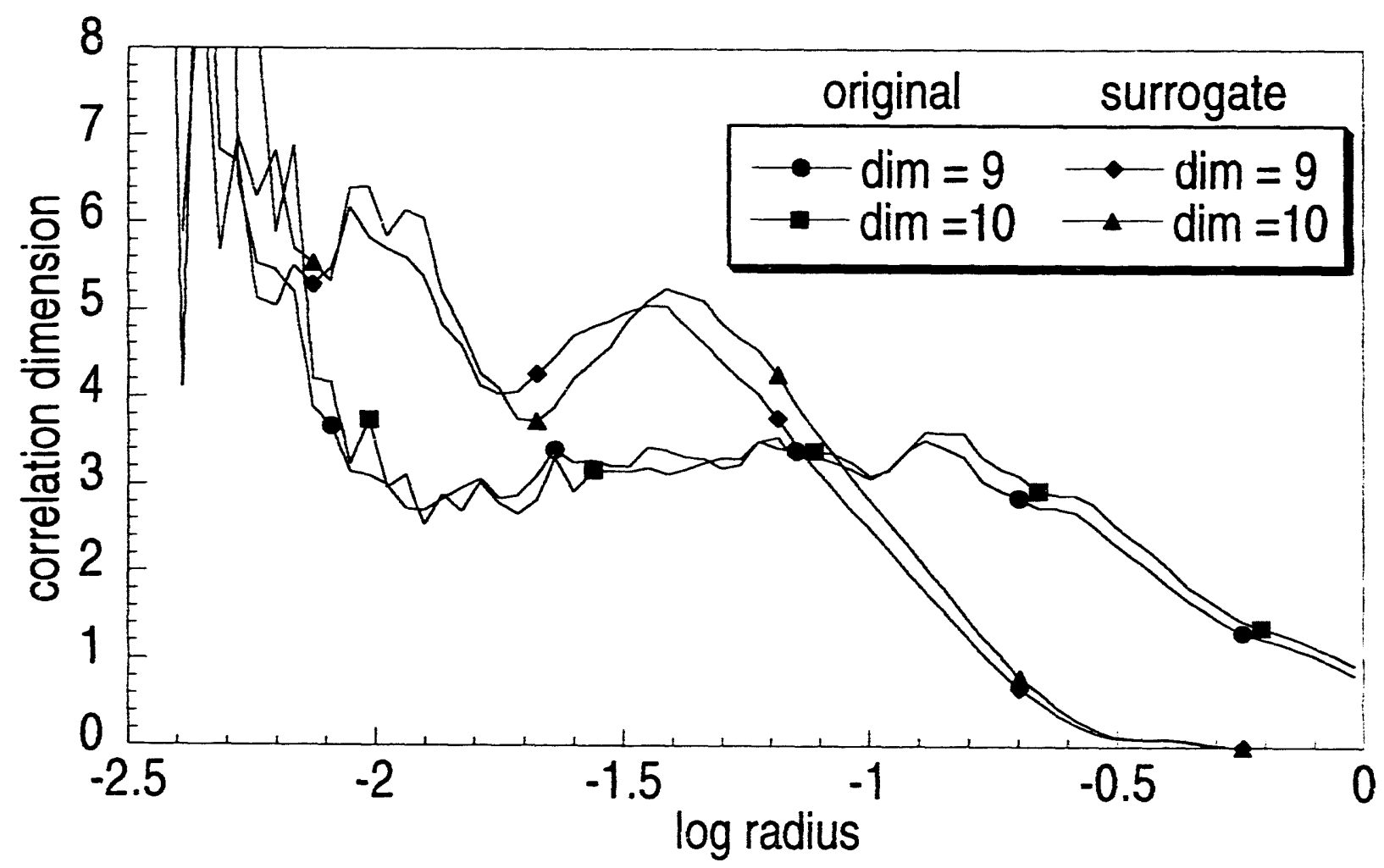

Fi: 2 


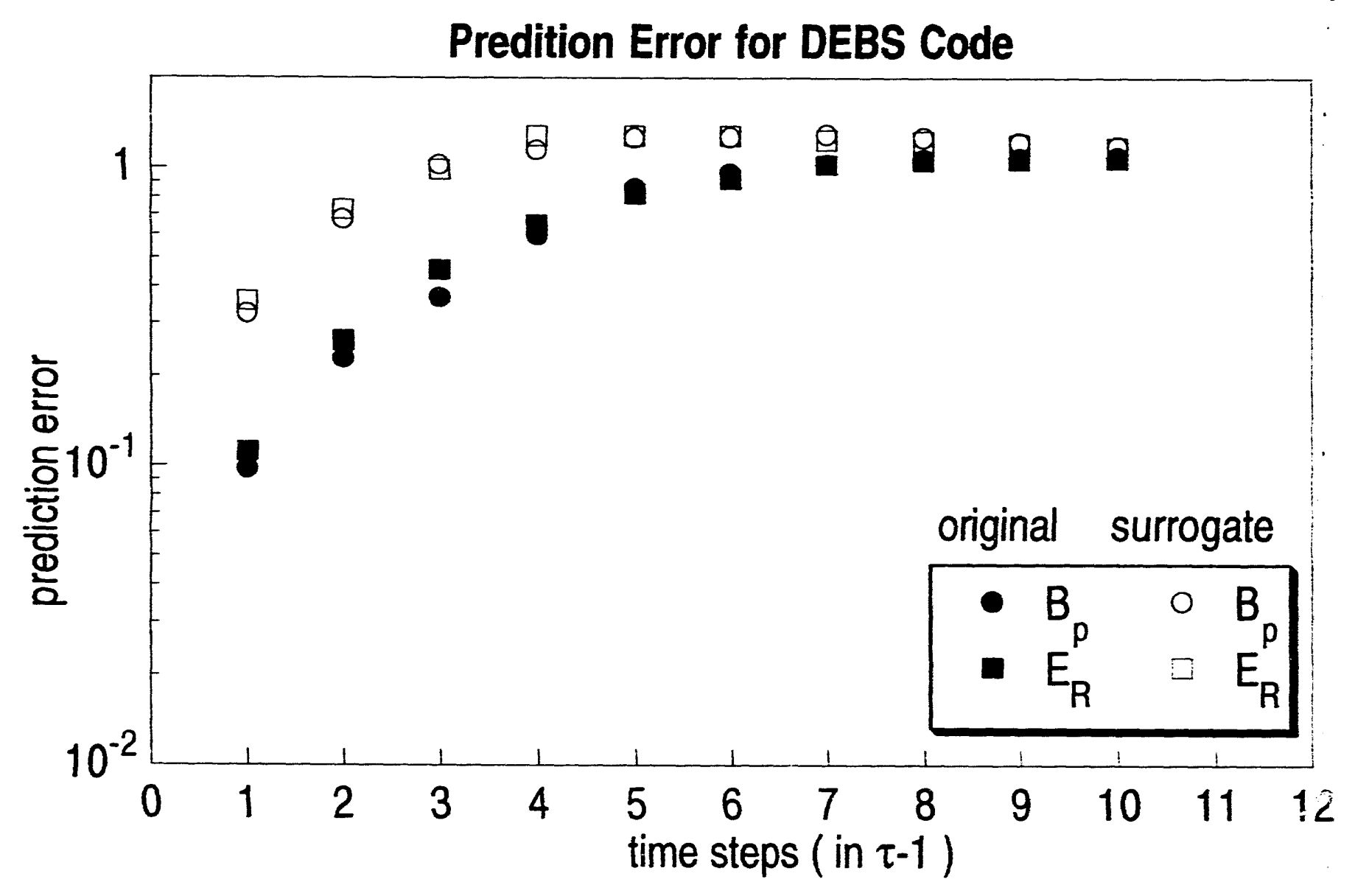

Fic 3 


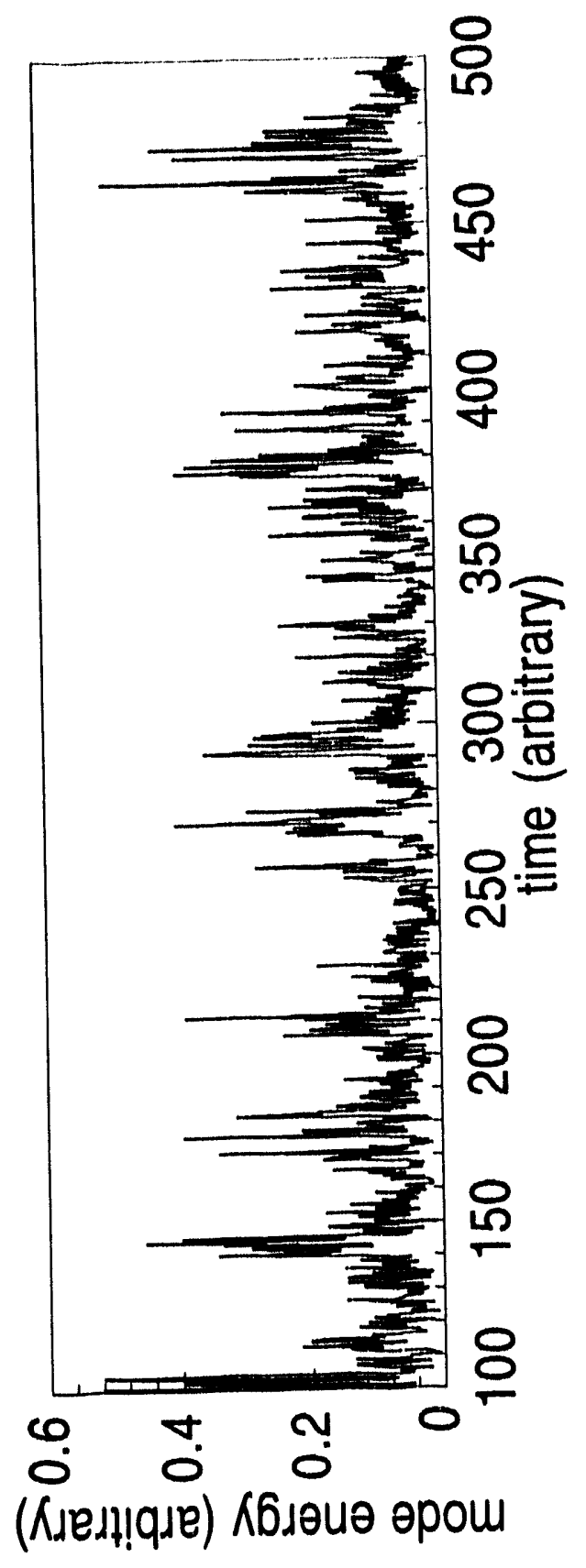




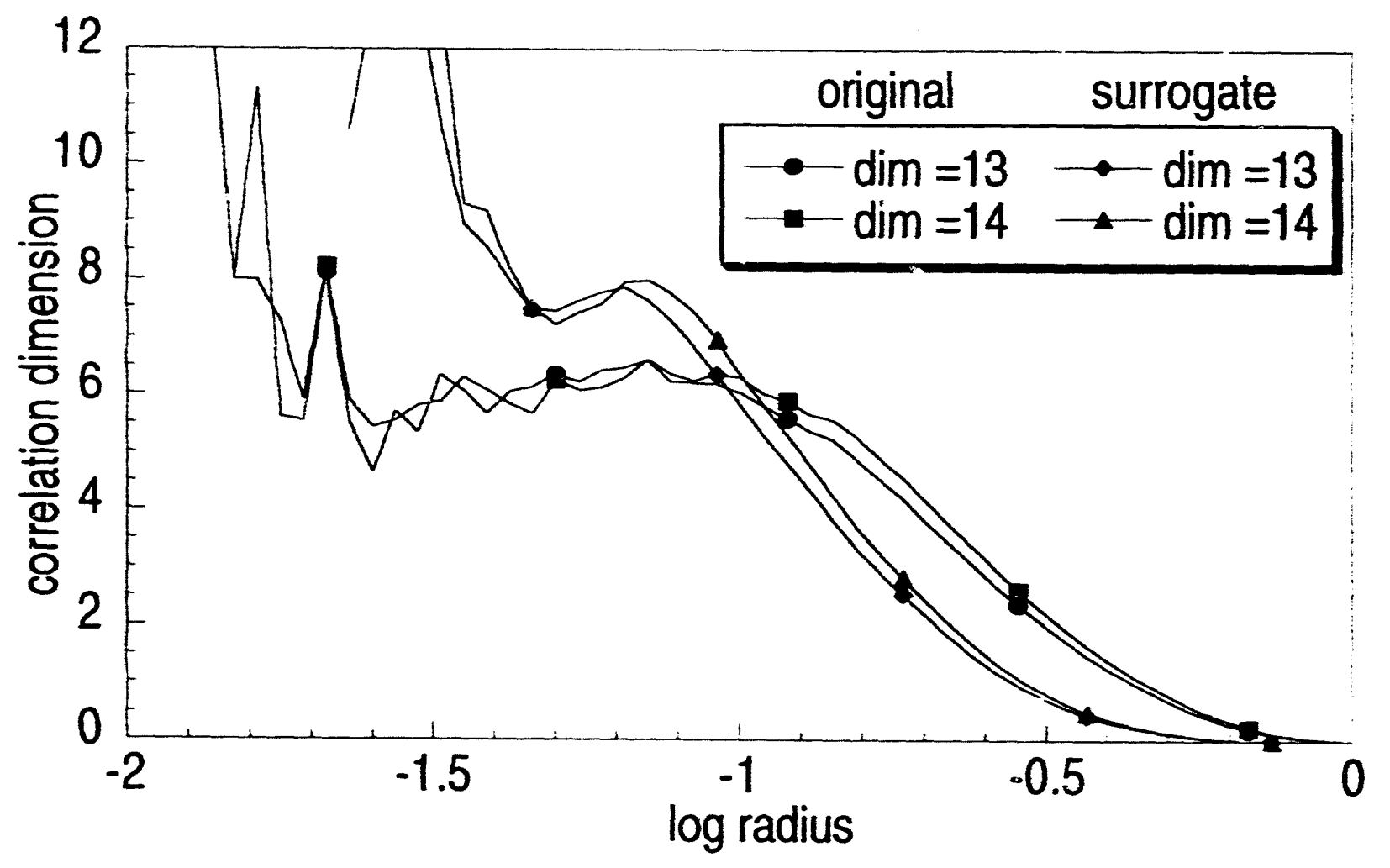

Fia 5 


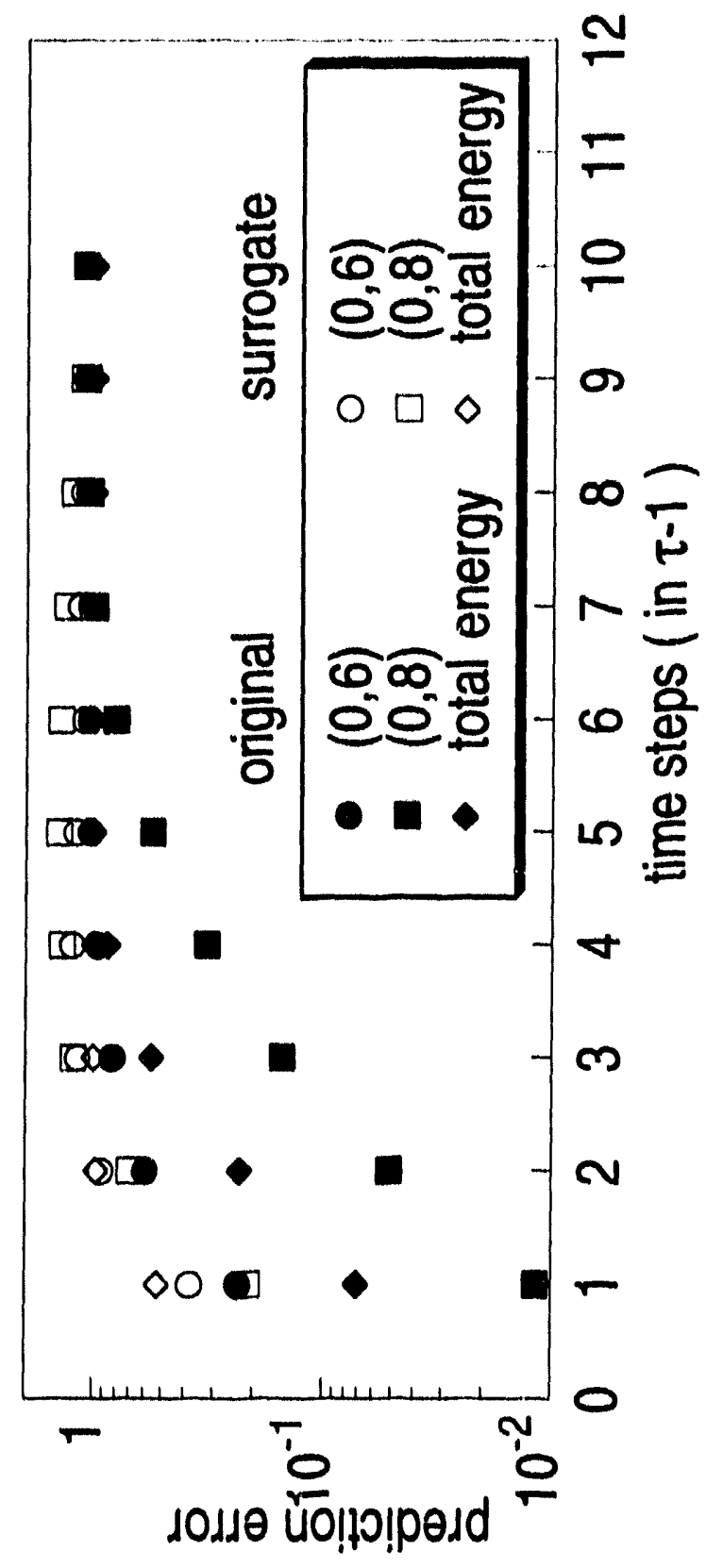




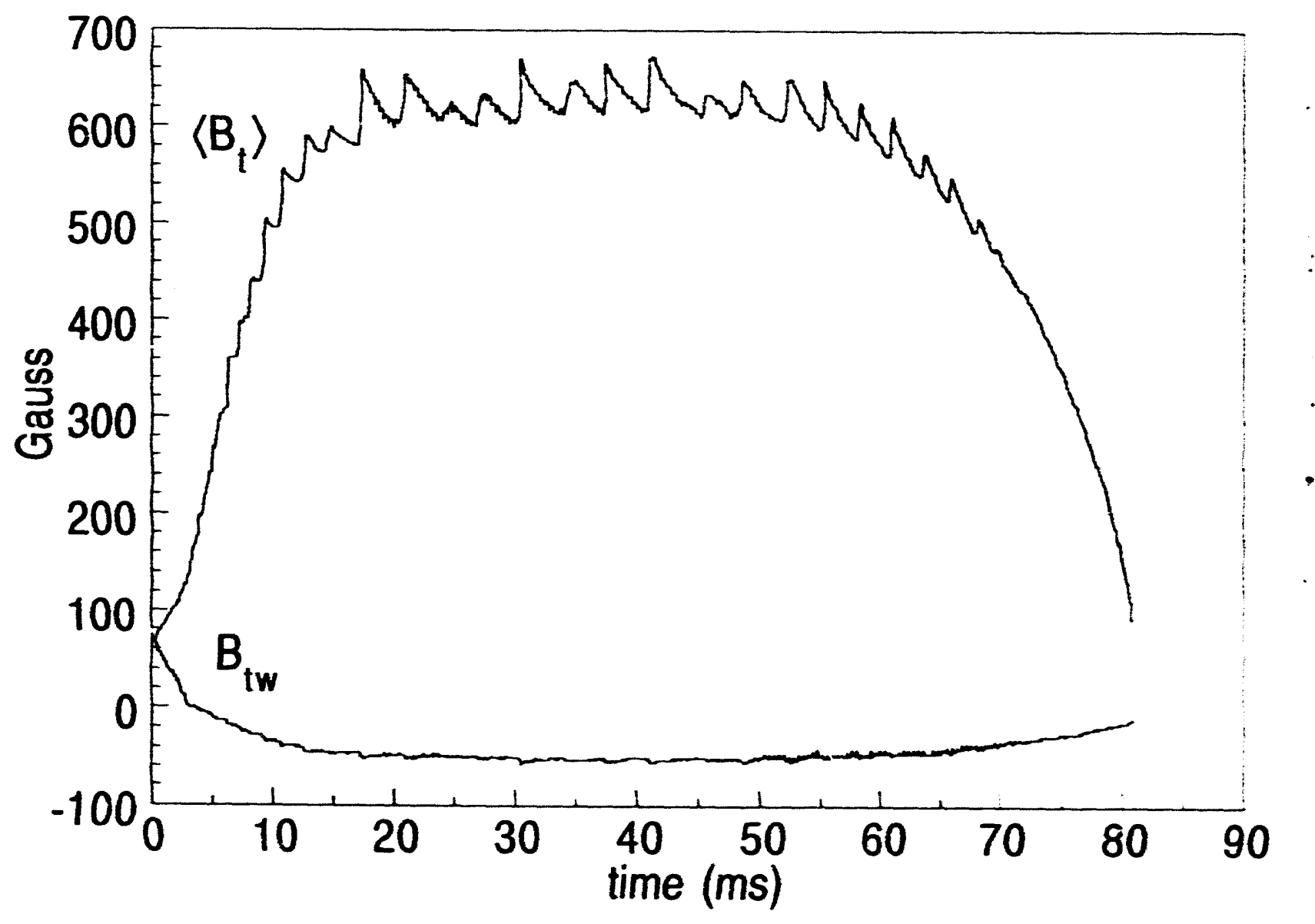




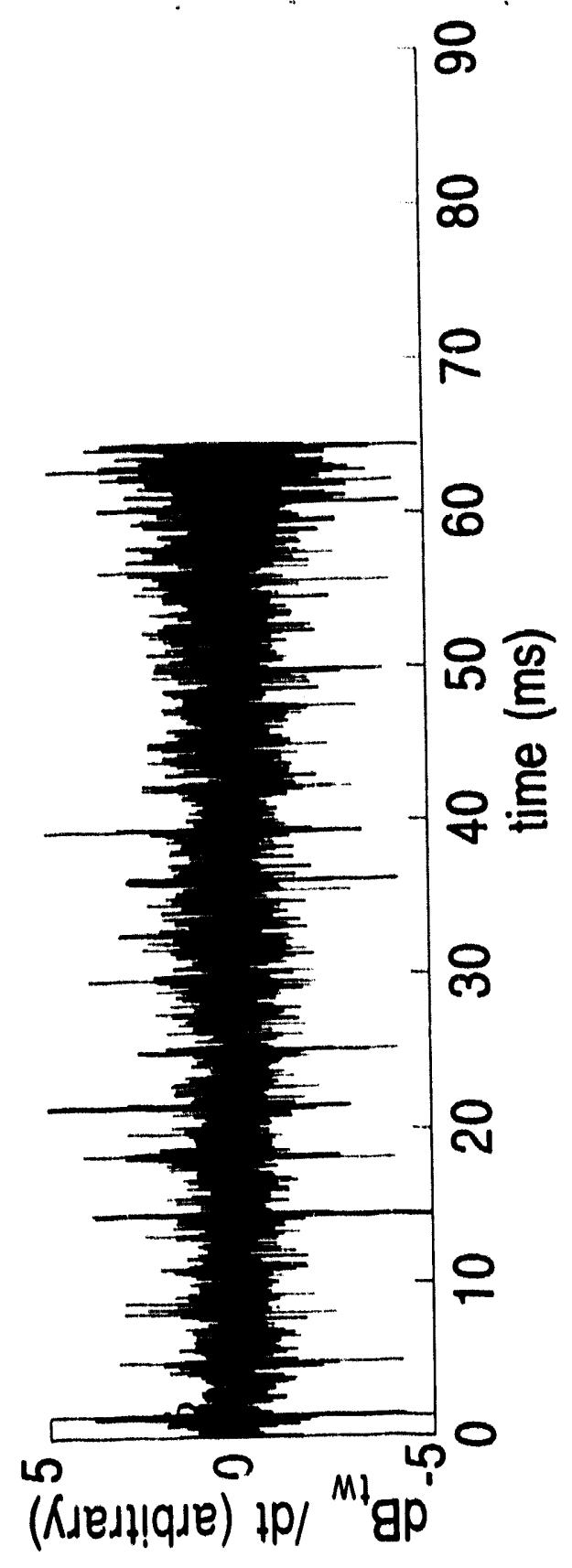

识

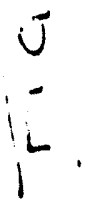




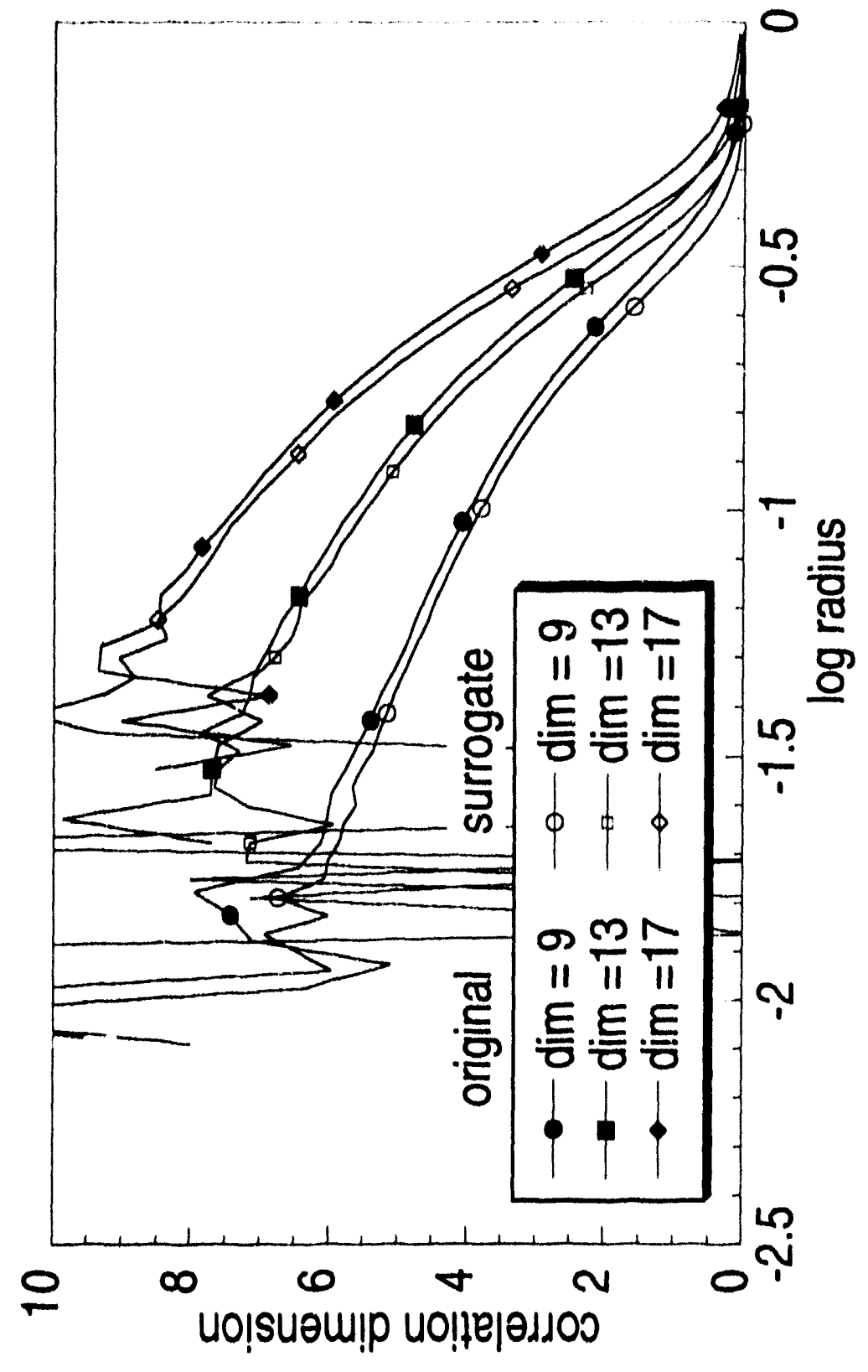




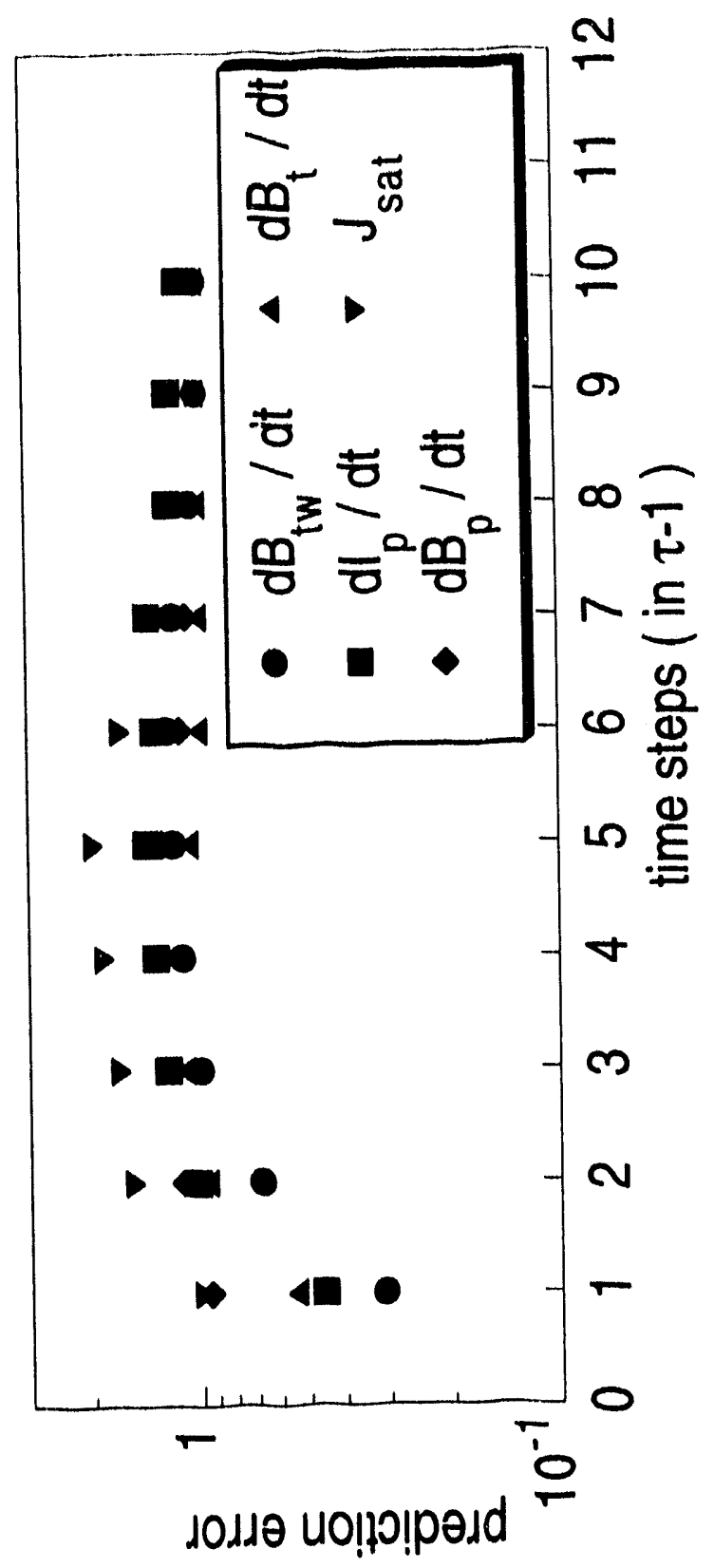




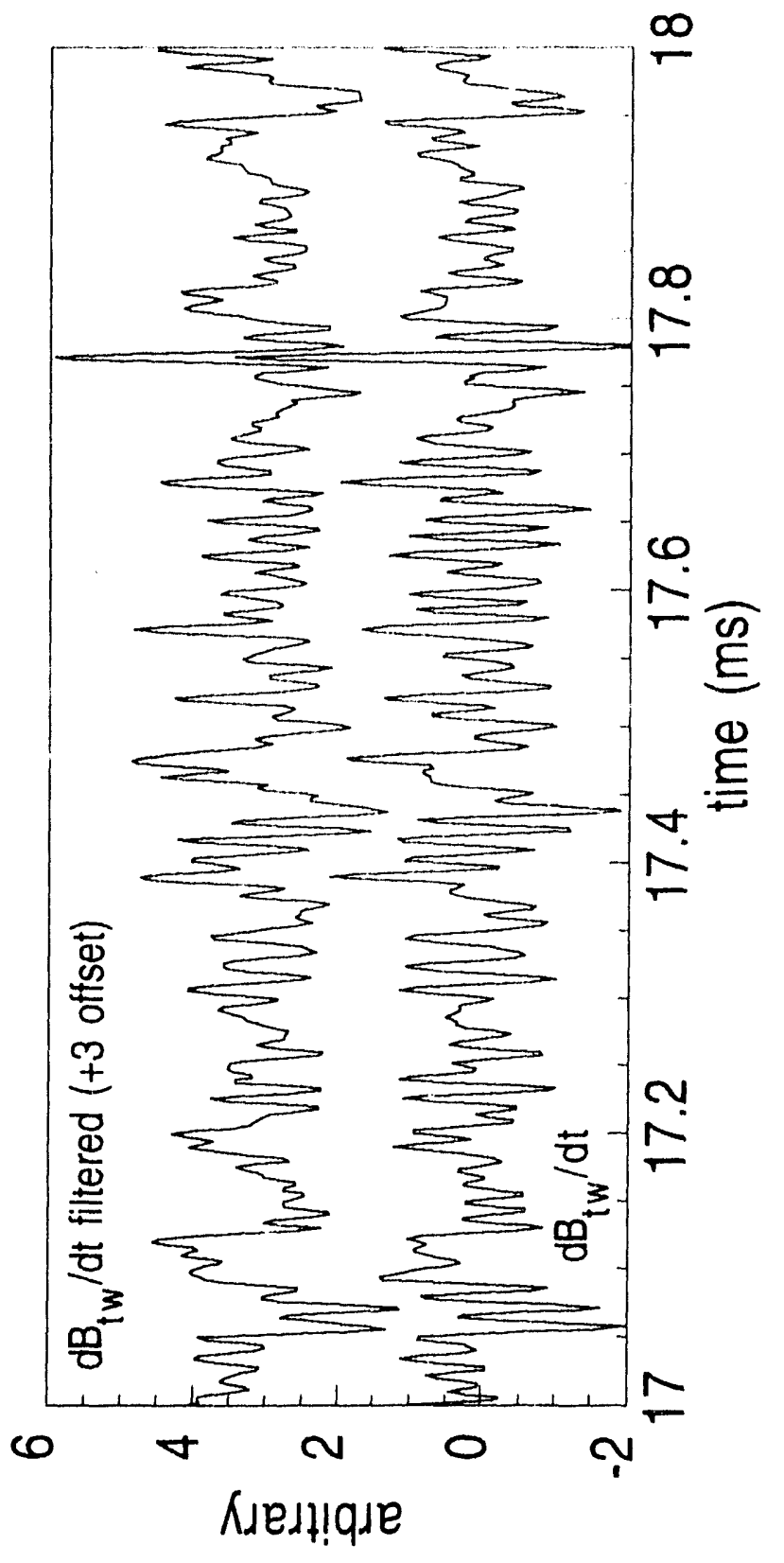




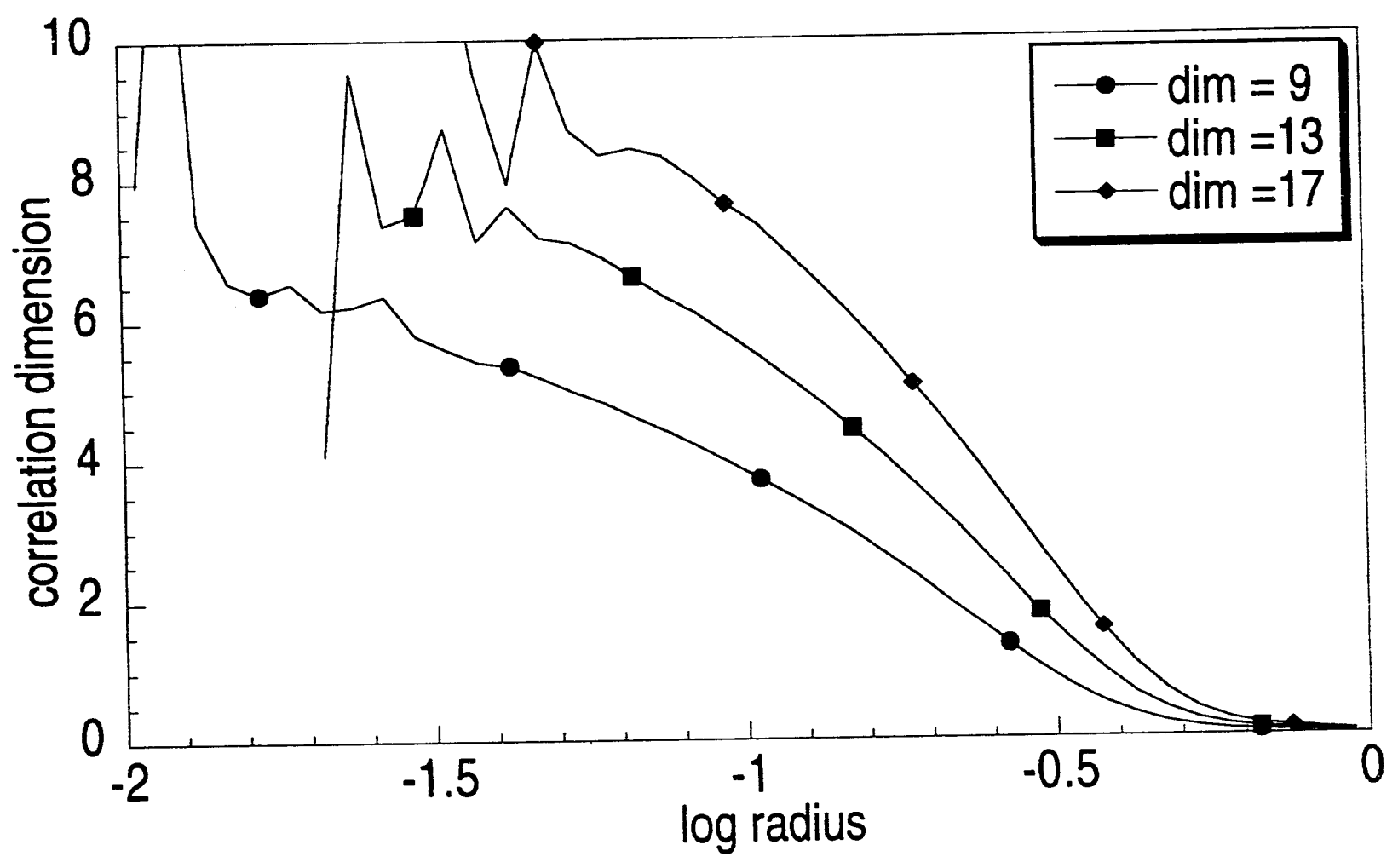

Fic 12 


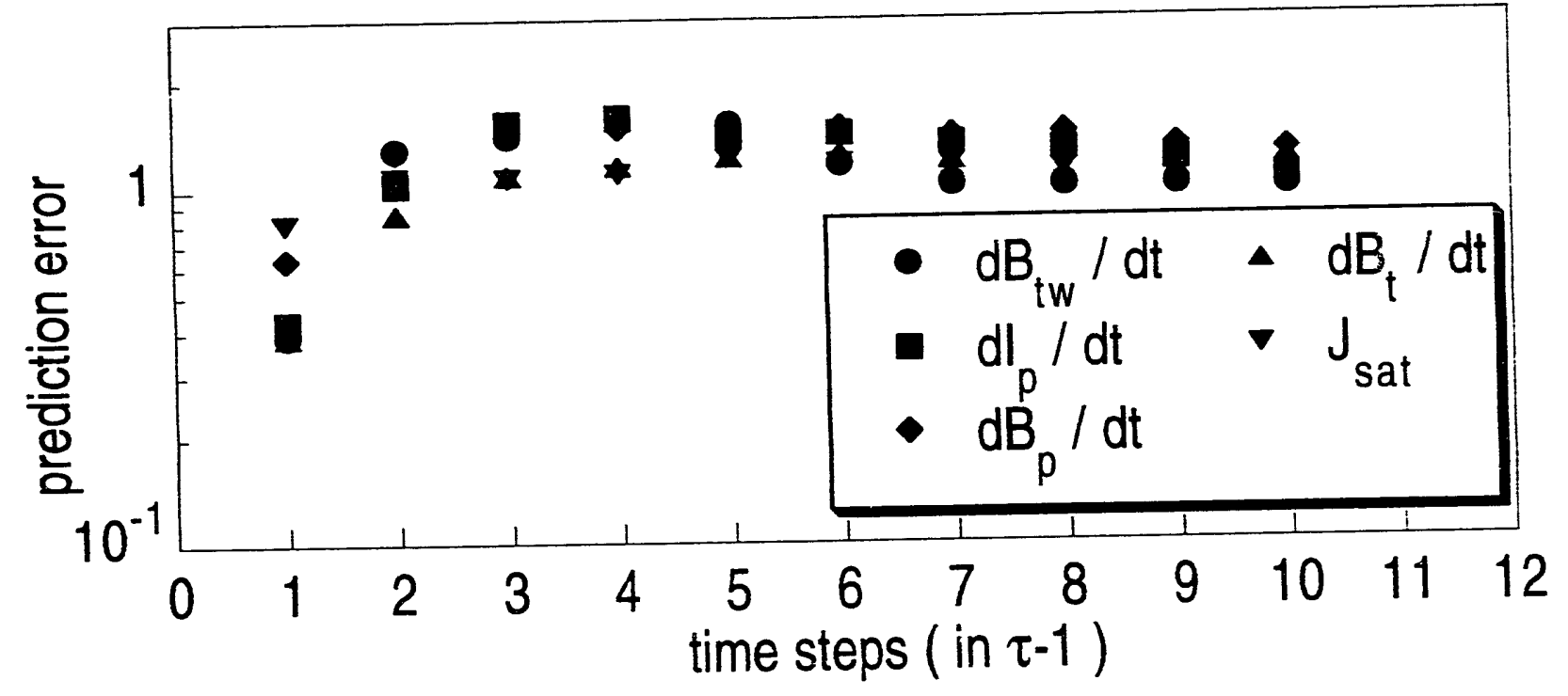

Fica 13 


\section{EXTERNAL DISTRIBUTION IN ADDITION TO LIC-20}

S.N. Rasband, Brigham Young University

T. Dolan, EG\&G Idaho, Inc.

R.A. Moyer, General Atomics

J.B. Taylor, Institute for Fusion Studies, The University of Texas at Austin

F. Uchimoto, University of Montana

F.W. Perkins, PPPL

O. Ishiharn, Texas Technical University

M.A. Abdou, University of California, Los Angeles

R.W. Conn, University of California, Los Angeles

P.E. Vandenplas, Association Euratom-Etat Belge, Belgium

Centro Brasileiro de Pesquisas Firicas, Brazil

[?. Sakanaka, Institute de Fisica-Unicamp, Brazil

Mme. Monique Bex, GANIL, France

J. Rader, CEN/CADARACHE, France

University of Ioannina, Greece

S. Ortolani, Istituto Gas Ionizzati, EURATON-ENEA-CNR Association, Italy

R. Andremi, Associazione EURA TOM-ENEA sulla Fusione, Italy

Plasma section, Energy Fundamentals Division Electrotechnical Laboratory, Japan

Y. Konduh, Gunma University, Kiryu, Gunma, Japan

H. Toyama, University of Tokyo, Japan

lOOM-Instituut voor Plasmafysica "Rijnhuizen," The Netherlands

Z. Ning, Academia Sinica, Peoples Republic of China

P. Yang, Shandongr University, Peoples Republic of China

S. Zhu, University of Science \& Technology of China, People's Republic of China I.N. Bogatu, Institute of Atomic Physics, Romania

M.J. Alport, University of Natal, Durban, South Africa

R. Storer, The Flinders University of South Australia, South Australia

B. I. chnert, Royal Institute of Technology, Sweden

Librarian, CRPP, Ecole Polytechnique Federale de Lausanne, Switzerland

B. Alper, Culham Laboratory, UK

A. Newton, UK

2 for Chicago Operations Office

4 for individuals in Washington Offices

INTERNAL DISTRIBUTION IN ADDITION TO UC-20

80 for local group and file 

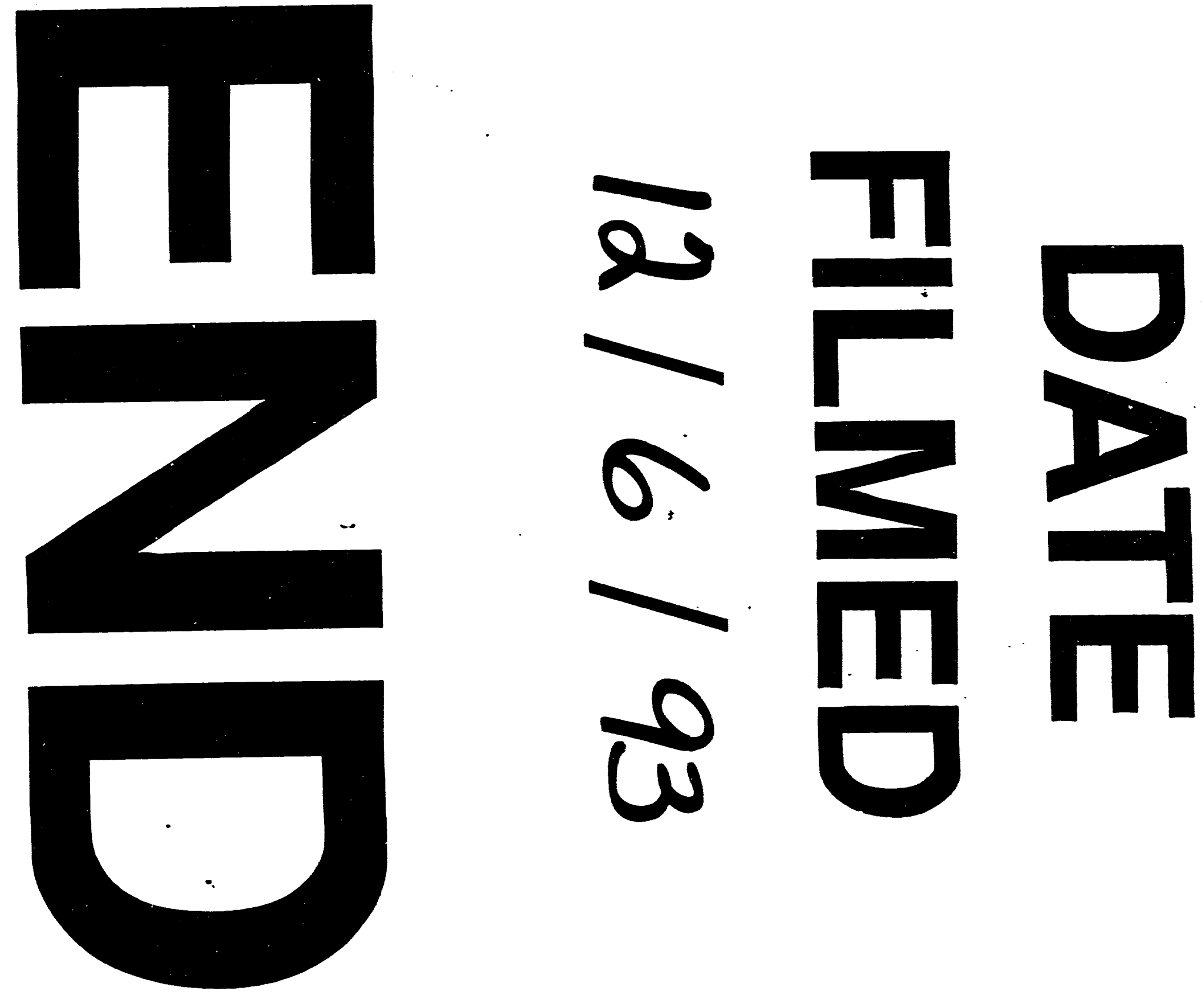
